\title{
On the orbital evolution of low mass protoplanets in turbulent, magnetised disks
}

\author{
R. P. Nelson
}

Astronomy Unit, Queen Mary, University of London, Mile End Rd, London E1 4NS, UK

e-mail: R.P.Nelson@qmul .ac.uk

Received 23 December 2004 / Accepted 11 August 2005

\section{ABSTRACT}

We present the results of MHD simulations of low mass protoplanets interacting with turbulent, magnetised protostellar disks. We calculate the orbital evolution of "planetesimals" and protoplanets with masses in the range $0 \leq m_{\mathrm{p}} \leq 30 M_{\oplus}$. The disk models are cylindrical models with toroidal net-flux magnetic fields, having aspect ratio $H / r=0.07$ and effective viscous stress parameter $\alpha \simeq 5 \times 10^{-3}$.

A significant result is that the $m_{\mathrm{p}}=0$ "planetesimals", and protoplanets of all masses considered, undergo stochastic migration due to gravitational interaction with turbulent density fluctuations in the disk. For simulation run times currently feasible (covering between 100-150 planet orbits), the stochastic migration dominates over type I migration for many models. Fourier analysis of the torques experienced by protoplanets indicates that the torque fluctuations contain components with significant power whose time scales of variation are similar to the simulation run times. These long term torque fluctuations in part explain the dominance of stochastic torques in the models, and may provide a powerful means of counteracting the effects of type I migration acting on some planets in turbulent disks. The effect of superposing type I migration torques appropriate for laminar disks on the stochastic torques was examined. This analysis predicts that a greater degree of inward migration should occur than was observed in the MHD simulations. This may be a first hint that type I torques are modified in a turbulent disk, but the results are not conclusive on this matter.

The turbulence is found to be a significant source of eccentricity driving, with the "planetesimals" attaining eccentricities in the range $0.02 \leq$ $e \leq 0.14$ during the simulations. The eccentricity evolution of the protoplanets shows strong dependence on the protoplanet mass. Protoplanets with mass $m_{\mathrm{p}}=1 M_{\oplus}$ attained eccentricities in the range $0.02 \leq e \leq 0.08$. Those with $m_{\mathrm{p}}=10 M_{\oplus}$ reached $0.02 \leq e \leq 0.03$. This trend is in basic agreement with a model in which eccentricity growth arises because of turbulent forcing, and eccentricity damping occurs through interaction with disk material at coorbital Lindblad resonances.

These results are significant for the theory of planet formation. Stochastic migration may provide a means of preventing at least some planetary cores from migrating into the central star due to type I migration before they become gas giants. The growth of planetary cores may be enhanced by preventing isolation during planetesimal accretion. The excitation of eccentricity by the turbulence, however, may act to reduce the growth rates of planetary cores during the runaway and oligarchic growth stages, and may cause collisions between planetesimals to be destructive rather than accumulative.

Key words. accretion, accretion disks - magnetohydrodynamics (MHD) - methods: numerical - planetary systems: formation planetary systems: protoplanetary disks

\section{Introduction}

The continuing discovery of extrasolar planets by the radial velocity and transit techniques has generated renewed interest in the theory of planet formation (e.g. Mayor \& Queloz 1995; Marcy et al. 2000; Vogt et al. 2002; Santos et al. 2003). In the so-called core instability model of planet formation, gas giant planets form through the build-up of a rocky and icy core of $\sim 15$ Earth masses, which then undergoes gas accretion resulting in a giant planet (e.g. Bodenheimer \& Pollack 1986; Pollack et al. 1996). The gas accretion stage of this process is normally believed to take a few million years, during which time the planetary cores slowly accrete gas through quasi-static settling, until the planet mass is around 50 Earth masses. Beyond this mass the gas accretion rate increases dramatically and gas is able to accrete onto the planet through a circumplanetary disk at the rate supplied by the protostellar disk (Papaloizou \& Nelson 2005). An alternative model for giant planet formation is that gravitational fragmentation of a fairly massive protostellar disk can form Jovian mass planets directly (e.g. Boss 2001). This model, however, is unable to account for terrestrial planets, and is unlikely to explain the existence of Uranus and Neptune.

Since giant planets form in a gas-rich protoplanetary disk, the gravitational interaction between planets and disks can play an important evolutionary role. This interaction has been the subject of many studies over the last couple of decades. In the usual picture, a protoplanet exerts torques on a 
protostellar disk through the excitation of spiral density waves at Lindblad resonances, and possibly through interaction at corotation resonance (e.g. Goldreich \& Tremaine 1979, 1980; Lin \& Papaloizou 1979; Papaloizou \& Lin 1984; Ward 1986, 1997; Tanaka et al. 2002). The spiral waves carry an associated angular momentum flux, which is deposited in the disk material where the waves damp, leading to exchange of angular momentum between protoplanet and disk. The disk lying exterior to the protoplanet orbit exerts a negative torque on the planet, and the interior disk exerts a positive torque. The negative outer disk torque usually dominates and the protoplanet migrates inward. This is referred to as type I migration. For protoplanets of $\sim 15$ Earth masses, the migration time is between $10^{4}$ and $10^{5} \mathrm{yr}$ (Tanaka et al. 2002), which is much shorter than the estimated gas accretion phase of giant planet formation (Pollack et al. 1996). Taken at face value, this presents a serious problem for the core-instability model of gas giant planet formation. In a recent paper, Papaloizou \& Nelson (2005) examined the possibility of shortening the formation time scale of gas giant planets by reducing the dust opacity in the outer radiative zone of the forming planets. Although the formation time can be shortened significantly, they concluded that the type I migration time is always shorter than the planet formation time. This analysis, however, pertains only to smooth, laminar disk models.

Until quite recently most models of viscous accretion disks used the Shakura \& Sunyaev (1973) $\alpha$ model for the anomalous disk viscosity. This assumes that the viscous stress is proportional to the thermal pressure in the disk, without specifying the origin of the viscous stress. Balbus \& Hawley (1991) showed that significant angular momentum transport in weakly magnetised disks could arise from the magnetorotational instability (MRI). Non linear numerical simulations performed using a local shearing box formalism (e.g. Hawley \& Balbus 1991; Hawley et al. 1996; Brandenburg et al. 1996) confirmed this and showed that the saturated non linear outcome of the MRI is MHD turbulence having an effective viscous stress parameter $\alpha$ of between $\sim 5 \times 10^{-3}$ and $\sim 0.1$, depending on the magnetic field configuration. Global simulations of MHD turbulent disks (e.g. Armitage 1998; Hawley 2000; Hawley 2001; Steinacker \& Papaloizou 2002; Papaloizou \& Nelson 2003) confirm the picture provided by the local shearing box simulations.

An obvious question is how the interaction of forming planets with protoplanetary disks changes when the disks are turbulent. Papaloizou \& Nelson (2003 - hereafter PN2003) examined and characterised the turbulence obtained in a variety of MHD disk models. Nelson \& Papaloizou (2003 hereafter NP2003) examined the interaction between a global cylindrical disk model and a massive ( 5 Jupiter masses) protoplanet. A similar study was undertaken by Winters et al. (2003). Papaloizou et al. (2004 - hereafter PNS2004) performed global cylindrical disk simulations and local shearing box simulations of turbulent disks interacting with protoplanets of different mass. The main focus of that paper was to characterise the changes in flow morphology and disk structure as a function of planet mass, and to examine the transition from linear to non linear interaction leading to gap formation. Nelson \& Papaloizou (2004 - hereafter NP2004) examined the migration of low and high mass protoplanets in turbulent disks by examining the time evolution of the torques exerted on the planets by the disks. They noticed that low mass planets experienced strongly varying torques due to interaction with the turbulent density fluctuations, and suggested that such planets would undergo stochastic migration rather than monotonic inward migration normally associated with type I migration in laminar disks. The planets, however, were maintained on fixed circular orbits and so their migration could not be examined directly.

In this paper we present the results of simulations of low mass protoplanets embedded in turbulent, magnetised disks, and allow the planet orbits to evolve due to interaction with the disk. In order to sample the range of outcomes, we consider a small ensemble of planets in each simulation. We find that in all simulations performed, the forces experienced by the protoplanet are highly variable, and as a result the protoplanet orbits evolve similarly to a random walk. A simple analysis suggests that the heavier planets we consider ought to undergo inward migration predominantly due to the underlying type I torques, but the simulations on the whole are not in agreement with this prediction. Fourier analysis of the torques experienced by the planets indicates that they experience stochastic forces with a broad range of associated time scales of variation, ranging from the planet orbital period to the simulation run time itself. These long time scale variations clearly contribute to the long term stochastic behaviour of the planets observed in the simulations. There is some evidence that the underlying type I migration is modified in turbulent disks, but this is not conclusive.

The results also indicate that MHD turbulence is able to drive significant growth of eccentricities for low mass objects. In particular, planetesimals and planets with masses similar to the Earth can obtain eccentricities $e \sim 0.1$. This clearly has potentially important consequences for planetary accumulation models. Heavier planets attain lower eccentricities apparently because their interaction with the disk at coorbital Lindblad resonances causes eccentricity damping (e.g. Artymowicz 1993; Papaloizou \& Larwood 2000).

The plan of the paper is as follows. In Sect. 2 we describe the governing equations. In Sects. 3 and 4 we describe the numerical method and the system of units used. The initial and boundary conditions used in the simulations are described in Sect. 5, and the simulation results are presented in Sect. 6. We discuss the simulation results in Sects. 7-9, focusing in particular on the issues of the balance between type I and stochastic migration, and the eccentricity evolution of planets and planetesimals. We summarise the paper and draw conclusions in Sect. 10.

\section{Equations of motion}

The equations of ideal MHD written in a frame rotating with uniform angular velocity $\Omega_{\mathrm{f}} \hat{z}$, with $\hat{z}$ being the unit vector along the rotation axis assumed to be in the vertical direction, are the continuity equation

$\frac{\partial \rho}{\partial t}+\nabla \cdot \rho \boldsymbol{v}=0$ 
the equation of motion

$\frac{\partial \boldsymbol{v}}{\partial t}+\boldsymbol{v} \cdot \nabla \boldsymbol{v}+2 \Omega_{\mathrm{f}} \hat{z} \times \boldsymbol{v}=-\frac{\nabla p}{\rho}-\nabla \Phi+\frac{(\nabla \times \boldsymbol{B}) \times \boldsymbol{B}}{4 \pi \rho}$

and the induction equation

$\frac{\partial \boldsymbol{B}}{\partial t}=\nabla \times(\boldsymbol{v} \times \boldsymbol{B})$

where $\boldsymbol{v}, P, \rho$, and $\boldsymbol{B}$ denote the fluid velocity, pressure, density and magnetic field respectively. The potential $\Phi=\Phi_{\text {rot }}+\Phi_{\mathrm{G}}$ contains contributions due to gravity, $\Phi_{\mathrm{G}}$, and the centrifugal potential $\Phi_{\text {rot }}=-(1 / 2) \Omega_{\mathrm{f}}^{2}|\hat{z} \times \boldsymbol{r}|^{2}$.

The gravitational potential has contributions from a central mass $M_{*}$ and $N$ planets with masses $m_{\mathrm{pi}}$. Thus in cylindrical coordinates $(r, \phi, z)$, with the planets located at $\left(r_{\mathrm{pi}}, \phi_{\mathrm{pi}}, 0\right)$ and the star located at the origin of the coordinate system, the gravitational potential is $\Phi_{\mathrm{G}}=\Phi_{\mathrm{c}}+\sum_{i=1}^{N} \Phi_{\mathrm{pi}}$, where

$\Phi_{\mathrm{c}}=-\frac{G M_{*}}{r}$

and

$\Phi_{\mathrm{pi}}=-\frac{G m_{\mathrm{pi}}}{\sqrt{r^{2}+r_{\mathrm{pi}}^{2}-2 r r_{\mathrm{pi}} \cos \left(\phi-\phi_{\mathrm{pi}}\right)+b^{2}}}$.

Here, as in the papers PN2003, NP2003, PNS2004 and NP2004, we have neglected the dependence of the gravitational potentials on $z$ along with the vertical stratification of the disk, for reasons of computational speed. Thus the simulations are of cylindrical disks (e.g. Armitage 1998, 2001; Hawley 2000, 2001; Steinacker \& Papaloizou 2002). To model the effects of the reduction of the planet potential with vertical height, we have incorporated a softening length $b$ in the potential.

We use a locally isothermal equation of state in the form

$P=c_{\mathrm{s}}(r)^{2} \cdot \rho$,

with $c_{\mathrm{s}}$ denoting the sound speed which is specified as a fixed function of the cylindrical radius $r$. Expressions (1)-(6) give the basic equations used for the simulations.

\subsection{Planet orbital evolution}

In this work we allow the planet orbits to evolve due to the gravitational forces they experience from the disk as well as the central star. For a single simulation we consider the evolution of either six or three planets concurrently, located at different positions within the disk. These planets do not interact with each other gravitationally, but affect each others evolution indirectly through perturbations they make to the disk structure. In the case of low mass planets, the effects of the turbulence will be of considerably greater significance.

The equation of motion for planet $i$ is:

$$
\frac{\mathrm{d} \boldsymbol{v}_{\mathrm{pi}}}{\mathrm{d} t}=-\frac{G M_{*}}{r_{\mathrm{pi}}^{3}} \boldsymbol{r}_{\mathrm{pi}}+\boldsymbol{f}_{\mathrm{di}}-2 \Omega_{\mathrm{f}} \hat{z} \times \boldsymbol{v}_{\mathrm{pi}}+\Omega_{\mathrm{f}} \hat{z} \times\left(\Omega_{\mathrm{f}} \hat{z} \times \boldsymbol{r}_{\mathrm{pi}}\right) .
$$

The acceleration due to the disk is given by

$\boldsymbol{f}_{\mathrm{di}}=-G \int_{V} \frac{\rho(\boldsymbol{r})\left(\boldsymbol{r}_{\mathrm{pi}}-\boldsymbol{r}\right)}{\left(r^{2}+r_{\mathrm{pi}}^{2}-2 r r_{\mathrm{pi}} \cos \left(\phi-\phi_{\mathrm{pi}}\right)+b^{2}\right)^{3 / 2}} \mathrm{~d} V$ where the integral is performed over the disk volume. Note that the planet potentials are also cylindrical in that there is no vertical component of gravity. During the simulations we monitor the torque per unit mass experienced by the planets, which is defined by

$\boldsymbol{T}_{i}=\boldsymbol{r}_{\mathrm{pi}} \times \boldsymbol{f}_{\mathrm{di}}$

Note that the torque per unit mass is independent of the planet mass, and so may be calculated for zero-mass "planetesimals" and for finite mass planets.

The calculations are performed in a uniformly rotating frame with angular velocity $\Omega_{\mathrm{f}}$. The planet equations of motion are evolved using a simple leap-frog integrator, with correct centering employed for inclusion of the Corioli's force.

\section{Numerical method}

The numerical scheme that we employ is based on a spatially second-order accurate method that computes the advection using the monotonic transport algorithm (Van Leer 1977). The MHD section of the code uses the method of characteristics constrained transport (MOCCT) as outlined in Hawley \& Stone (1995) and implemented in the ZEUS code. The code has been developed from a version of NIRVANA written originally by U. Ziegler (Ziegler \& Yorke 1997).

\section{Computational units}

We use units in which the central mass $M_{*}=1$, the gravitational constant $G=1$, and the radius $R=1$ corresponds to the radial location of the inner boundary of the computational domain. The unit of time is $\Omega^{-1}=\sqrt{G M_{*} / R^{3}}$, although we report our results in units of the orbital period at the disk inner edge, which we denote as $P(1)=2 \pi \Omega^{-1}$. Planets are positioned in the disk between radii $r_{\mathrm{pi}}=2.2-3.2$. We note that the orbital period at $r_{\mathrm{pi}}=2.2$ is $P(2.2)=3.26 P(1)$ and at $r_{\mathrm{pi}}=3.2 \mathrm{we}$ have $P(3.2)=5.72 P(1)$. Simulation runs times are typically $\simeq 500 P(1)$, corresponding to $\sim 153 P(2.2)$ and $\sim 90 P(3.2)$. If we take the computational radius $r=2.5$ to correspond to $5 \mathrm{AU}$, then a simulation run time of $500 P(1)$ corresponds to $\sim 1335 \mathrm{yr}$, for a solar mass central star.

\section{Initial and boundary conditions}

The simulations presented here all use the same underlying turbulent disk model. This model has a constant aspect ratio $H / r \equiv c(r) /(r \Omega)=0.07$, where $\Omega$ is the disk angular velocity measured in the inertial frame. The inner radial boundary of the computational domain is at $R_{\text {in }}=1$ and the outer boundary is at $R_{\text {out }}=5$. The simulations were performed in the rotating frame with $\Omega_{\mathrm{f}}=0.28668$, which is the Keplerian angular velocity at a radius of $r=2.3$.

The boundary conditions employed are very similar to those described in PN2003. Regions of the disk in the vicinity of the inner and outer boundaries were given non-Keplerian angular velocity profiles (uniform in the simulations described here) that are stable to the MRI, and which have large values of 
the density in order to maintain radial hydrostatic equilibrium. These regions act as buffer zones that prevent the penetration of magnetic field to the radial boundaries, thus maintaining the initial value of net magnetic flux in the computational domain. The inner buffer zone runs from $r=1.2$ to $r=R_{\text {in }}$. The outer buffer zone runs from $r=4.5$ to $r=R_{\text {out }}$.

The creation of a relaxed turbulent disk model in which planets could be immersed to examine their orbital evolution was constructed using a multi-stage procedure. This is because the initial relaxation of a turbulent disk model can significantly modify the initial surface density structure, due to radial and temporal variations in the magnetic stresses during the relaxation. It is desirable to minimise this effect so that a reasonably smooth profile is used for the disk in which the planets are inserted. Also, we wish to obtain a turbulent disk model in which the volume averaged $\alpha$ value is $\simeq 5 \times 10^{-3}$, as expected for protostellar disks, and the magnetic field strength in the disk was adjusted to achieve this.

The calculations presented in NP2004 used an initial setup where $R_{\text {in }}=1$ and $R_{\text {out }}=8$. The numerical resolution used was greater than that used here $\left(\left[N_{r}, N_{\phi}, N_{z}\right]=[450,1092,44]\right.$, as opposed to $[264,608,44]$ used in this paper), but those simulations could only be run for $\sim 25$ planet orbits. The higher resolution used in NP2004 meant that zero net flux magnetic fields could be used, requiring a self-sustaining dynamo to maintain the field. The lower resolution adopted for the simulations in this paper leads to the requirement that net flux magnetic fields be used to sustain the turbulence over long time periods. This is because the lower resolution simulations cannot maintain an active turbulent state for zero net flux fields. The use of net flux fields guarantees the continued existence of magnetic field in the simulation domain, which helps maintain the turbulence. A modest magnetic flux is used to obtain a disk with modest turbulent stresses.

In order to obtain the desired disk model, the following procedure was adopted:

(i) The disk was initiated with a toroidal magnetic field in a finite annulus in the disk between the radii $1.5 \leq r \leq 4$. The initial field varied as $B_{\phi}(r)=B_{0} / r$, and $B_{0}$ was defined such that the initial ratio of volume integrated magnetic pressure to thermal pressure was $1 /\langle\beta\rangle=2.73 \times 10^{-3}$ (where $\beta=8 \pi P / B^{2}$ ). The initial density distribution in the disk was $\rho(r)=\rho_{0} / r$ away from the buffer zones, with $\rho_{0}$ defined such that the disk model would contain approximately $0.06 M_{\odot}$ interior to $40 \mathrm{AU}$ (assuming $r=2.5$ corresponds to $5 \mathrm{AU}$ ). In other words, the basic model is chosen to be around three times more massive than the minimum mass solar nebula model. The disk model was relaxed for a period of 191.7 orbits at the disk inner boundary.

(ii) The disk model was restarted at this point with the initial density distribution re-established, but with all other quantities remaining the same after the disk was relaxed for 191.7 orbits. The model was then run for a further 58.3 orbits.

(iii) The model was restarted with the initial density distribution re-established, and with the magnetic field strength being reduced throughout the disk by a factor of $1 / \sqrt{2}$. The model was run for a further 37.2 orbits.

(iv) The model was restarted after a further reduction in the magnetic field strength by a factor of $1 / \sqrt{2}$, and with the initial density profile having been re-established. The model was relaxed for a further 32 orbits. The final model has a volume averaged $\langle\alpha\rangle=5.04 \times 10^{-3}$, and $1 /\langle\beta\rangle=$ 0.012 , which are similar to those obtained in the models presented in PN2003, PNS2004 and NP2004.

The disk model has a full $2 \pi$ azimuthal domain, and vertical domain from $z_{\min }=-0.14$ to $z_{\max }=0.14$. Periodic boundary conditions were employed in the vertical and azimuthal directions.

Gravitational softening of the protoplanet was employed in all simulations, with the softening parameter $b=0.33 H$ and $H$ being evaluated at each planet position.

\section{Simulation results}

\subsection{Turbulent stresses}

In order to describe average properties of the turbulent models, we use quantities that are both vertically and azimuthally averaged over the $(\phi, z)$ domain (e.g. Hawley 2000). The vertical and azimuthal average of $Q$ is defined through

$\overline{Q(r, t)}=\frac{\int \rho Q \mathrm{~d} z \mathrm{~d} \phi}{\int \rho \mathrm{d} z \mathrm{~d} \phi}$

The average is taken over the full $2 \pi$ in azimuth. The disk surface density is given by

$\Sigma=\frac{1}{2 \pi} \int \rho \mathrm{d} z \mathrm{~d} \phi$

The vertically and azimuthally averaged Maxwell and Reynolds stresses are defined as follows:

$T_{\mathrm{M}}(r, t)=2 \pi \Sigma \overline{\left(\frac{B_{r}(r, \phi, z, t) B_{\phi}(r, \phi, z, t)}{4 \pi \rho}\right)}$

and

$T_{\mathrm{Re}}(r, t)=2 \pi \Sigma \overline{\delta v_{r}(r, \phi, z, t) \delta v_{\phi}(r, \phi, z, t)}$.

Here the velocity fluctuations $\delta v_{r}$ and $\delta v_{\phi}$ are defined through,

$\delta v_{r}(r, \phi, z, t)=v_{r}(r, \phi, z, t)-\overline{v_{r}}(r, t)$

$\delta v_{\phi}(r, \phi, z, t)=v_{\phi}(r, \phi, z, t)-\overline{v_{\phi}}(r, t)$.

The Shakura \& Sunyaev (1973) $\alpha$ stress parameter appropriate to the total stress is defined by

$\alpha(r, t)=\frac{T_{\mathrm{Re}}-T_{\mathrm{M}}}{2 \pi \Sigma \overline{(P / \rho)}}$, 
$\langle\alpha\rangle$ versus time

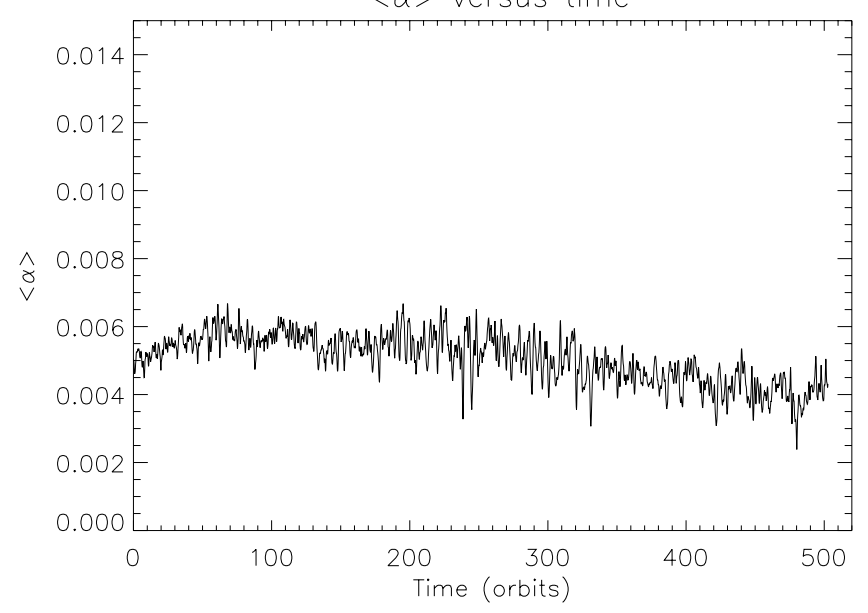

Fig. 1. This figure shows the time evolution of the volume averaged total $\alpha$ value in the disk. Note that the time is expressed in units of the orbital period at the inner edge of the disk.

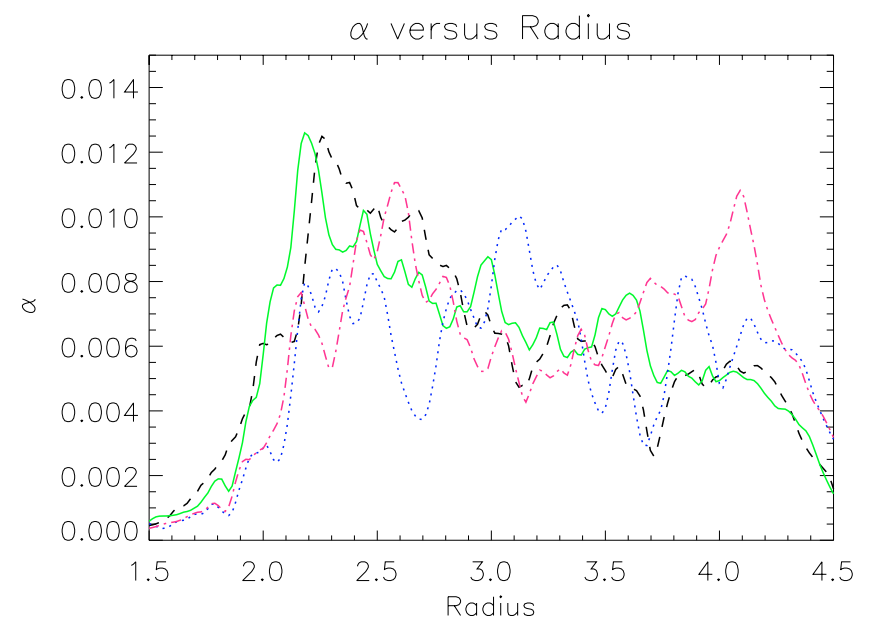

Fig. 2. This figure shows the evolution of the radial variation in the total $\alpha$ value in the disk. The solid line corresponds to a time of 15.6 orbits at the inner disk edge, the dashed line corresponds to 31.4 orbits, the dotted line to 71.2 orbits, and the dash-dotted line to 188.1 orbits.

\subsection{Disk model}

The method used to set up the turbulent disk model is described in Sect. 5. The subsequent evolution of the volume averaged $\alpha$ value is shown in Fig. 1, for the duration of the simulation performed with zero-mass planets described below. Values of the volume averaged stress parameter $\langle\alpha\rangle \simeq 5 \times 10^{-3}$ are sustained throughout the duration of the simulation, which is approximately 500 orbits at the disk inner edge. Snapshots of the radial distribution of $\alpha$ are shown in Fig. 2, showing that a magnetically active disk is sustained, at least for radii beyond $r \geq 2$.

Figure 3 shows snapshots of the midplane density for the model with six $10 M_{\oplus}$ protoplanets embedded. These images show that the density perturbations induced by planets of this mass and lower are exceeded by those generated by the turbulence. The consequences of this on the planet orbital evolution are described below for planet masses ranging between $0 \leq m_{\mathrm{p}} \leq 30 M_{\oplus}$. The effects of the turbulence on protoplanet orbital evolution are essentially stochastic, and for this reason the simulations were performed with six or three planets in order to sample the range of possible outcomes.

\subsection{Zero mass protoplanets - "planetesimals"}

The orbital evolution of the six "zero-mass planets" is shown in Fig. 4. These planets began with orbital radii $r_{\mathrm{pi}}=2.2,2.4,2.6$, 2.8, 3.0, 3.2 and randomised azimuthal positions. From now on we shall refer to these zero-mass objects as planetesimals, as they represent the evolution of bodies whose gravity is too weak to substantially perturb the disk structure, and for which no underlying type I migration occurs. We note that planetesimals experience gas drag, which is able to modify their orbits. We have neglected this effect here, but models of planetesimal evolution including gas drag will be presented in a future paper.

The left hand panel of Fig. 4 shows the evolution of the planetesimals' semimajor axes, with each line representing the evolution of a different planetesimal. It is clear from this plot that the gravitational interaction of the planetesimals with the turbulent density fluctuations causes the semimajor axes to change stochastically. This results in the planetesimals undergoing a "random walk" in orbital location, with variations of the semimajor axis at the 5-10 percent level being observed over time periods corresponding to $\sim 100$ planetesimal orbits.

The right hand panel shows the evolution of the planetesimal eccentricities. Not surprisingly the effect of the turbulence is to excite significant orbital eccentricity in the planetesimal population. Typical values obtained are $e \simeq 0.03$, but with values ranging from $0.01 \leq e \leq 0.13$ over the run times of $\simeq 100$ planetesimal orbits.

The results presented in Fig. 4 suggest that MHD turbulence will have a significant effect on the orbital evolution of planetesimals in protoplanetary disks, which will in turn have important repercussions for models of planet formation. In particular, the effects of stochastic migration will help to prevent the isolation of forming planetary embryos, and reduce the effects of orbital repulsion and planetesimal shepherding during oligarchic growth (Ida \& Makino 1993; Kokubo \& Ida 1998; Thommes et al. 2003). This may allow the growth of planetary cores to proceed to the $10-15 M_{\oplus}$ range, required for the formation of giant planets via the core instability scenario (e.g. Pollack et al. 1996). However, it is also clear that MHD turbulence may hinder the growth of planetary cores by exciting significant orbital eccentricities of the planetesimals, reducing the effects of gravitational focusing. For planetesimals in the $1-10 \mathrm{~km}$ range, it is unlikely that gas drag will be effective in reducing these eccentricities because of the dominant contribution from the turbulence as a source of excitation. Under these conditions an alternative source of eccentricity damping may be required, such as inelastic collisions between planetesimals, leading also to fragmentation and more effective gas drag for the smaller fragments. Of course, it then remains an open question how planetesimals can form in the first place in such a turbulent environment. This may be related to the existence of "dead zones" in which the ionisation fraction is too low to support MHD-driven turbulence (e.g. Gammie 1996). 

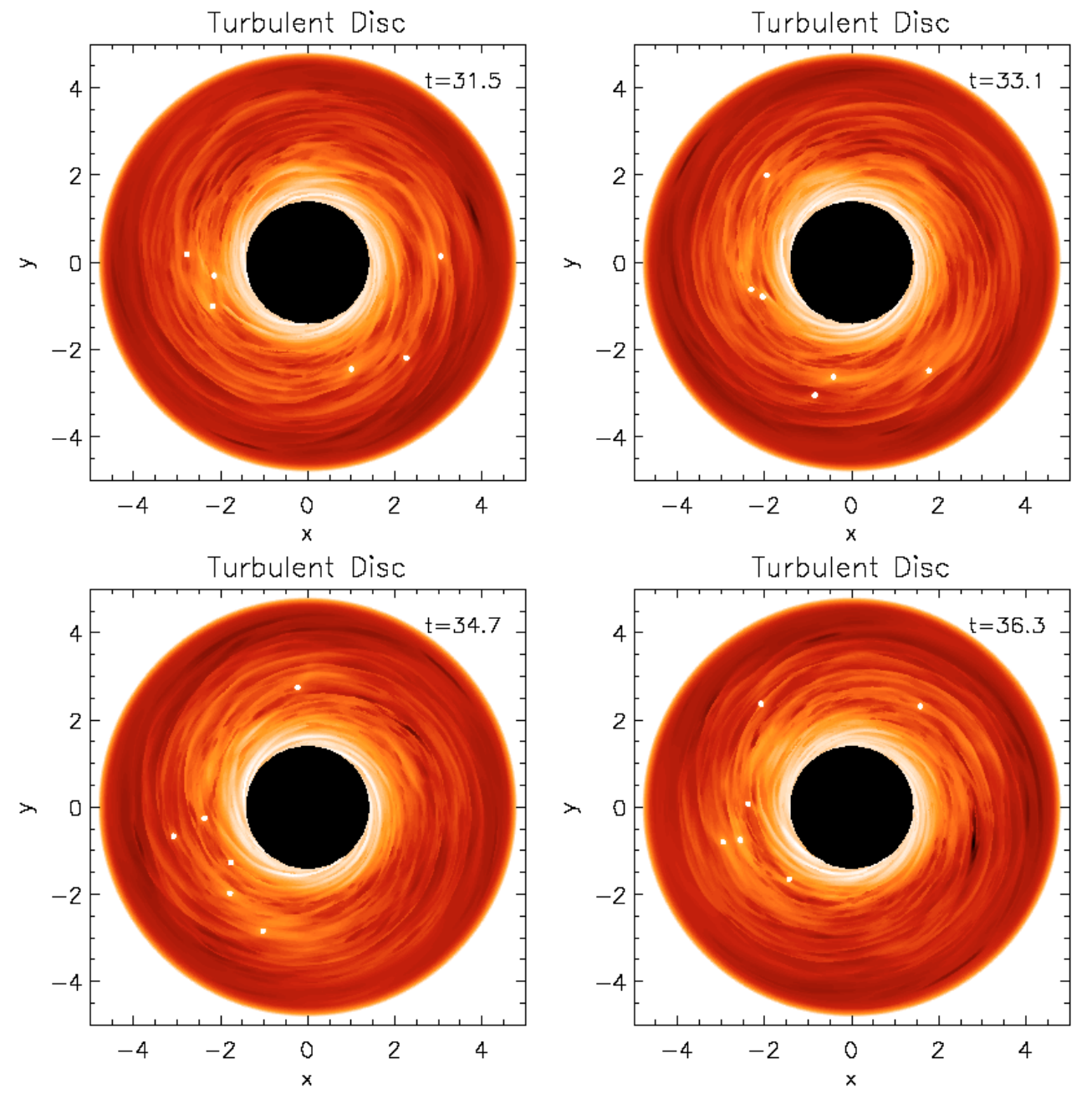

Fig. 3. This figure shows snapshot images of the disk midplane density for the run with $m_{\mathrm{p}}=10 M_{\oplus}$ planets. It is clear that the turbulent density fluctuations are typically larger than the spiral wakes generated by the planets in this case. The times corresponding to each image are shown at the top right of each panel, in units of the orbital period at the disk inner edge.

\subsection{Finte mass planets}

The simulations presented in the next five sections (6.5-6.9) were performed for either six or three planets placed in turbulent disks. These planets do not interact with each other gravitationally, as the purpose of these simulations is to sample the effects of turbulence on single planets. The planets may, however, affect each other indirectly through their influence on the disk. For planets whose orbits are well separated, this influence appears to be insignificant compared to the turbulence. In some simulations, however, there are planets that approach one another and occassionally cross orbits, increasing the mutual indirect influence. Such simulations will no longer be sampling the effects of MHD turbulence on isolated planets. We note that this issue is likely to be most important for the more massive planets.

The planets in the simulation involving the $30 M_{\oplus}$ objects do not approach each other. The simulation including the $10 M_{\oplus}$ protoplanets does involve close encounters. We have examined the torques experienced by the planets, along with their semimajor axis and eccentricity evolution, and can detect no obvious sign that the evolution is being modified by the close encounters. We are confident that the main results of this paper are not contaminated by this effect.

\section{5. $m_{\mathrm{p}}=1$ Earth mass protoplanets}

Figure 5 shows the orbital evolution of the $1 M_{\oplus}$ protoplanets. The dotted lines in the left panel show the evolution of planet orbits for simulations in which six $1 M_{\oplus}$ planets were evolved in a laminar disk model that was otherwise identical to the turbulent disk model. The solid lines, corresponding to planets in the turbulent disk, show similar stochastic migration as observed for the planetesimals. Indeed the protoplanet initially located at $r_{\mathrm{p}}=2.4$ migrates inward for $\sim 70$ orbits measured at the inner disk edge at first, before migrating outward for a sustained period of $\sim 130$ orbits, modifying its semimajor axis by almost 20 percent during this time. The other planets are also seen to migrate in a quasi-random fashion, but over less extreme distances.

The evolution of the eccentricities is plotted in the right hand panel. A tendency for eccentricity growth to significant values for some of the protoplanets is observed, in line with the results for the planetesimals. It is interesting to observe 

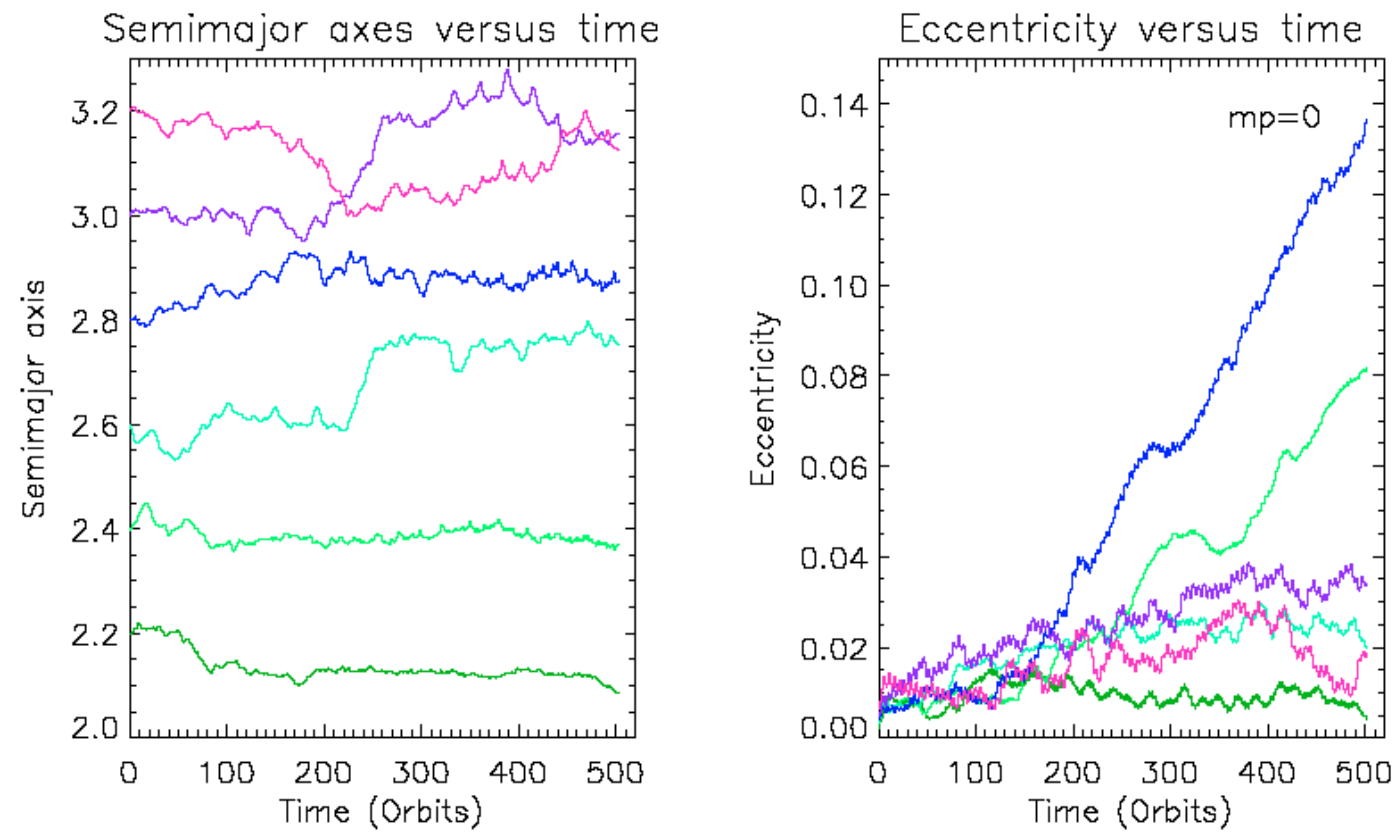

Fig. 4. This figure shows the evolution of the planet semimajor axes (left panel) and eccentricities (right panel) for the run with planet mass set to zero. It is clear from this plot that the time varying gravitational field of the disk causes stochastic migration of the "planetesimals", and significant eccentricity growth.
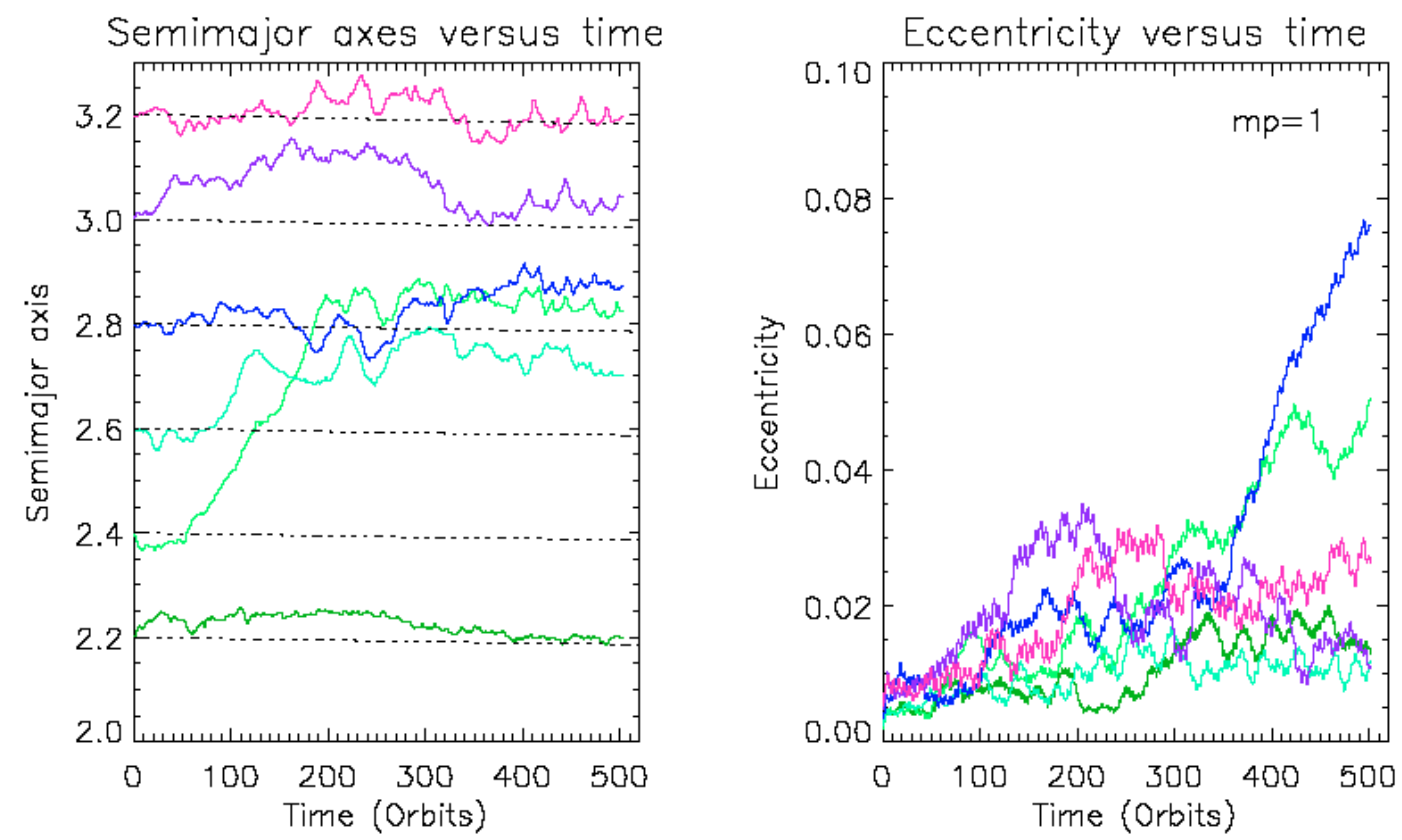

Fig. 5. This figure shows the evolution of the planet semimajor axes (left panel) and eccentricities (right panel) for the run with $m_{\mathrm{p}}=1 M_{\oplus}$. The dotted lines show the evolution of planet orbits for simulations in which six $1 M_{\oplus}$ planets were evolved in a laminar disk model that was otherwise identical to the turbulent disk model. The migration rates obtained are in quite good agreement with the rates predicted by Papaloizou \& Larwood (2000) for planets with softened potentials migrating in laminar disks. The solid lines represent the planets in the turbulent disk, and show that the planets undergo migration similar to a random walk for the duration of the simulation, with no clear tendency for the planets to migrate inward or outward.

however, that the peak eccentricities obtained are somewhat less than those obtained in the planetesimal cases, suggesting that the gravitational interaction with the disk is providing eccentricity damping to counteract the effects of the turbulent excitation. This is a trend that continues as the protoplanet mass increases, as described in the following sections.

\section{6. $m_{p}=3$ Earth mass protoplanets}

The orbital evolution of the $3 M_{\oplus}$ protoplanets is illustrated in Fig. 6. The left panel shows a similar evolution for the semimajor axes as has been described for the planetesimals and $1 M_{\oplus}$ protoplanets. The semimajor axes are again observed to 

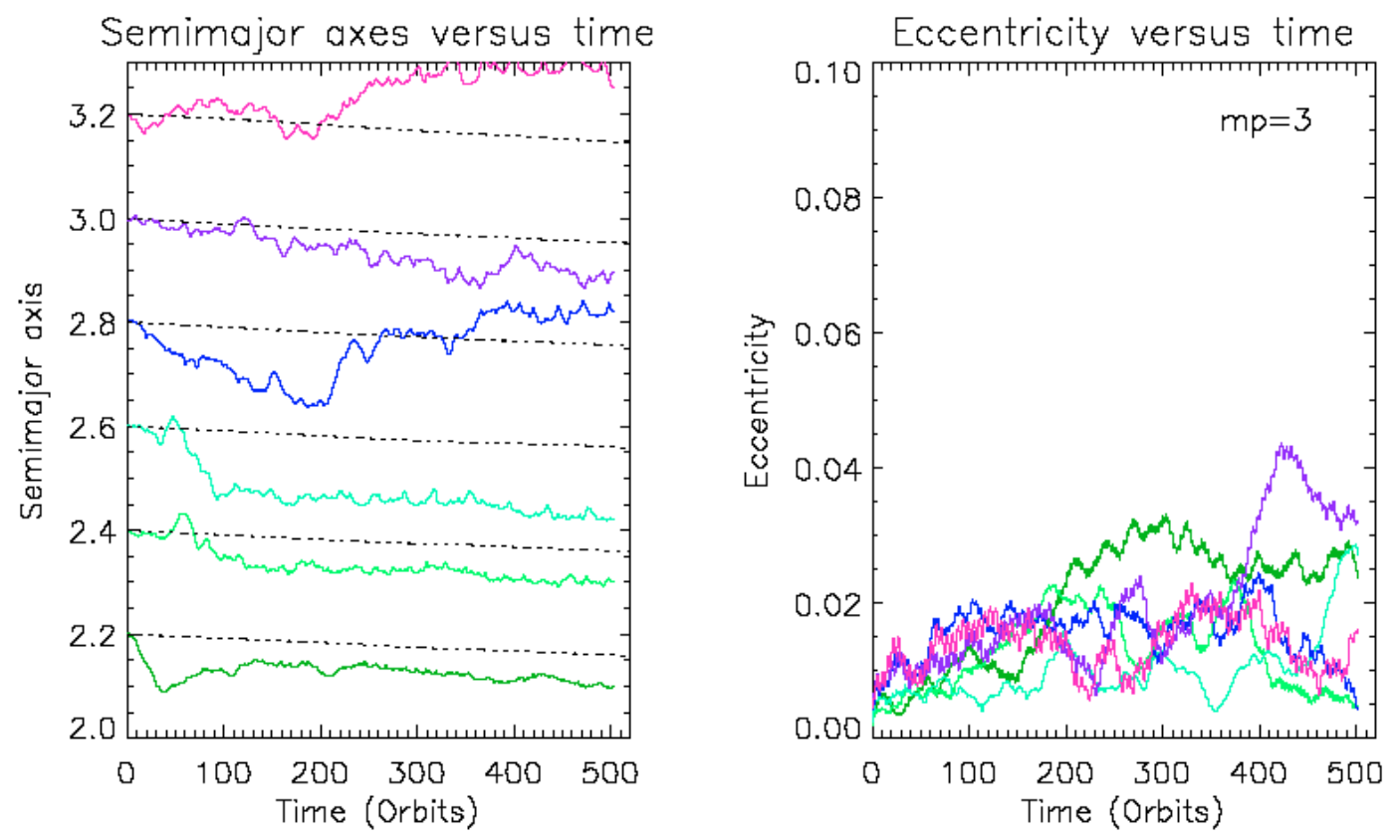

Fig. 6. This figure shows the evolution of the planet semimajor axes (left panel) and eccentricities (right panel) for the run with $m_{\mathrm{p}}=3 M_{\oplus}$. The figures show that the planets undergo migration similar to a random walk for the duration of the simulation, with no clear tendency for the planets to migrate inward or outward.

undergo modification in a stochastic manner, with variations at the 5 percent level being observed. The dotted lines show the results of a simulation in which six $3 M_{\oplus}$ protoplanets were evolved in a laminar disk. The monotonic inward migration of each of these planets is clearly discernible, suggesting that the planets do not greatly influence each others interaction with the disk. Inward migration in this case occurs close to the expected type I rate. The simulated torques are smaller than those presented in Papaloizou \& Larwood (2000) by a factor of 0.71 .

The eccentricity evolution is illustrated in the right panel of Fig. 6. Significant variations in the eccentricities are again observed, with peak values of $e \simeq 0.05$ being excited. It is interesting to compare the evolution with the planetesimal and $1 M_{\oplus}$ cases, as there is a clear trend toward obtaining lower eccentricities with increasing planet mass. It is likely that the eccentricity evolution is determined by a balance between stochastic forcing due to the turbulence, and eccentricity damping due to interaction with the disk at coorbital Lindblad resonances (Goldreich \& Tremaine 1980; Artymowicz 1993). This point is examined and discussed further in Sect. 7.

\section{7. $m_{\mathrm{p}}=5$ Earth mass protoplanets}

The orbital evolution of the six $5 M_{\oplus}$ protoplanets is shown in Fig. 7. The semimajor axes in the left panel show a general inward trend, which might at first be thought to be evidence that the usual type I migration is becoming dominant over the stochastic forcing as the planet mass increases. Closer inspection of the figures shows that the protoplanets are still subject to substantial random forcing. Furthermore the $10 M_{\oplus}$ case discussed below suggests that the general inward motion of the planets in Fig. 7 is probably a statistical effect rather than evidence for type I migration overwhelming stochastic forcing. It is worth noting, however, that for all cases considered in which the planets have masses $m_{\mathrm{pi}}>1 M_{\oplus}$, the inner most planet migrates inward at a rate similar to or greater that the expected type I rate. This arises because the planet moves into a region of the disk where it is less turbulent due to the initial set up, and where the density increases during the course of the simulation due to disk accretion.

The eccentricity evolution shown in the right panel of Fig. 7 continues to show the trend of lower eccentricity for higher mass planets. Here the peak value for the eccentricity obtained is $e \simeq 0.04$, but with eccentricities in the range $0.01 \leq e \leq 0.02$ being more typical.

\section{8. $m_{p}=10$ Earth mass protoplanets}

Figure 8 shows the orbital evolution of the six $10 M_{\oplus}$ protoplanets. Examination of the left panel shows that the planets' orbital evolution remains dominated by stochastic forcing for this mass, with the semimajor axes of three of the planets showing net increases for the duration of the simulation. This suggests that the general inward motion for the $5 M_{\oplus}$ planets seen in Fig. 7 is a statistical effect. The dotted lines in the left panel of Fig. 8 show the evolution of six $10 M_{\oplus}$ planets in a laminar disk. The expected inward migration is observed for each planet close to the predicted rate (Papaloizou \& Larwood 2000), showing that the planets have little effect on each others orbital evolution due to their perturbation of the disk.

The eccentricity evolution is shown in the right panel of Fig. 8. It is clear that the eccentricities remain quite small in this case, with peak values reaching $e \simeq 0.025$. More generally, however, the eccentricities remain at $\simeq 0.01$. The overall trend of eccentricity with planet mass remains such that considerably less eccentricity growth is observed for higher mass 

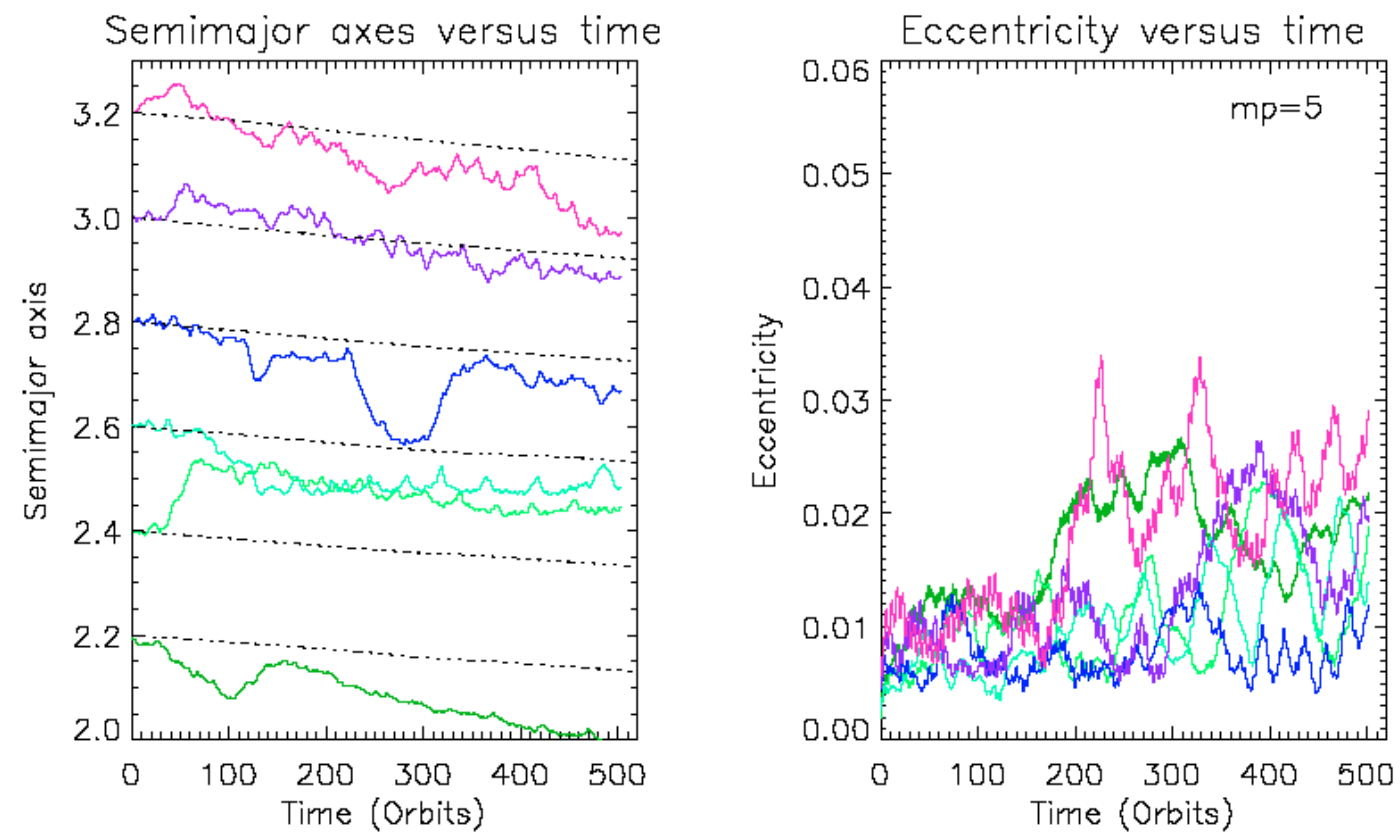

Fig. 7. This figure shows the evolution of the planet semimajor axes (left panel) and eccentricities (right panel) for the run with $m_{\mathrm{p}}=5 M_{\oplus}$. The figures show a general inward trend for the migration, but this appears to be a statistical fluke as the $m_{\mathrm{p}}=10 M_{\oplus}$ cases do not. Note that the scale on the $y$ axis of the right panel has changed compared with that in similar Figs. 4-6.
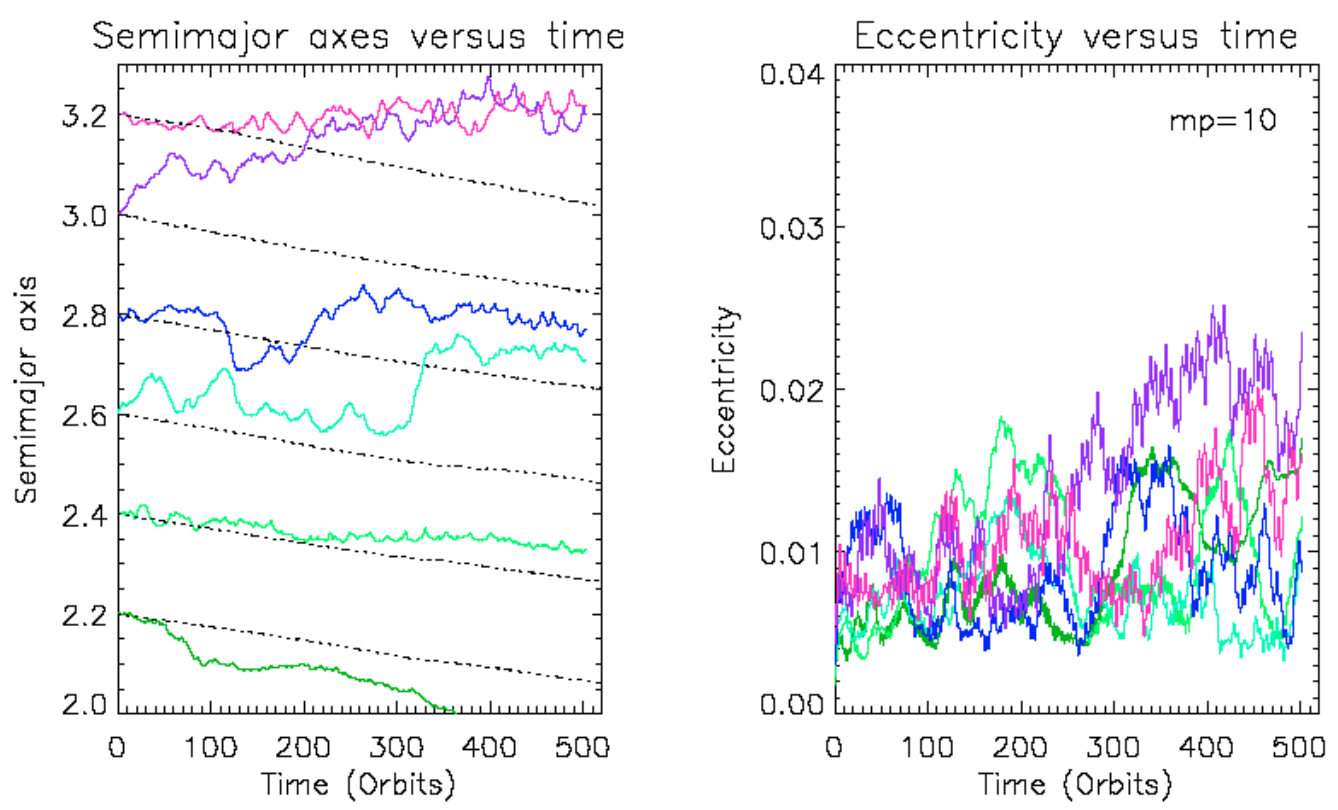

Fig. 8. This figure shows the evolution of the planet semimajor axes (left panel) and eccentricities (right panel) for the run with $m_{\mathrm{p}}=10 M_{\oplus}$. The figures show that the planets undergo migration similar to a random walk for the duration of the simulation, with no clear tendency for the planets to migrate inward or outward. Note that the scale on the $y$ axis of the right panel has changed compared with that in similar Figs. 4-7.

planets. The indication is that disk-planet interaction at coorbital Lindblad resonances causes eccentricity damping, and for planet masses of $m_{\mathrm{pi}} \sim 10 M_{\oplus}$ this dominates over turbulent forcing.

\section{9. $m_{p}=30$ Earth mass protoplanets}

We consider the orbital evolution of three $30 M_{\oplus}$ protoplanets, in contrast to the six planets considered for smaller mass objects, since they exert larger perturbations on the disk. Snapshots of the midplane density distribution are shown in Fig. 9, which show that the planets are now massive enough to excite density waves of similar amplitude to the background turbulent fluctuations. A similar trend was noted by NP2004.

The semimajor axis evolution is shown in the left panel of Fig. 10. The inner most planet is observed to migrate inward, such that after 400 orbits it has reached the same location as predicted for a laminar disk. This is again related to the fact that the inner disk is less turbulent. The outer two planets, however, 

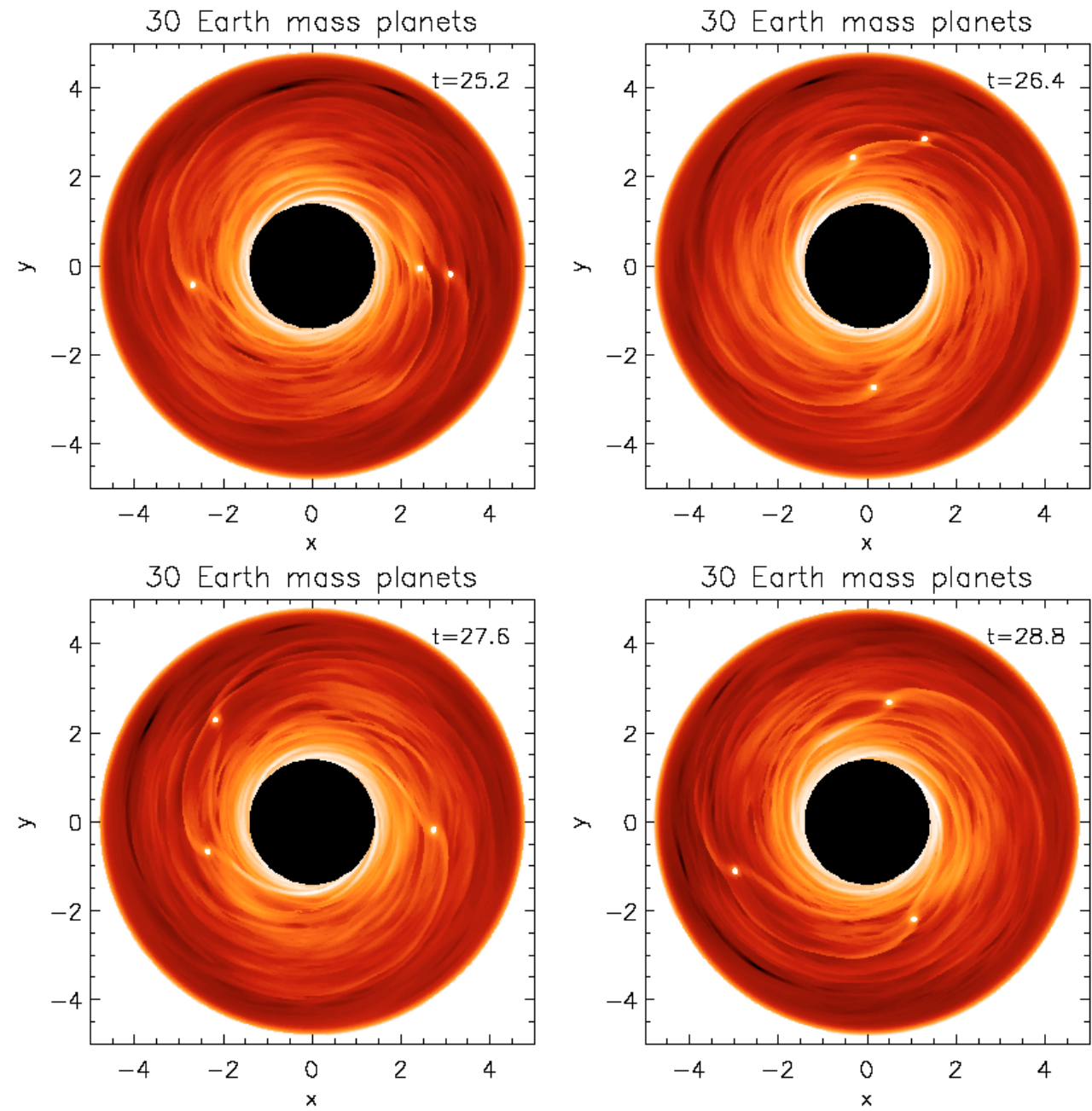

Fig. 9. This figure shows snapshot images of the disk midplane density for the run with $m_{\mathrm{p}}=30 M_{\oplus}$ planets. It is clear that the turbulent density fluctuations are of similar amplitude to the spiral wakes generated by the planets in this case. The times corresponding to each image are shown at the top right of each panel, in units of the orbital period at the disk inner edge.
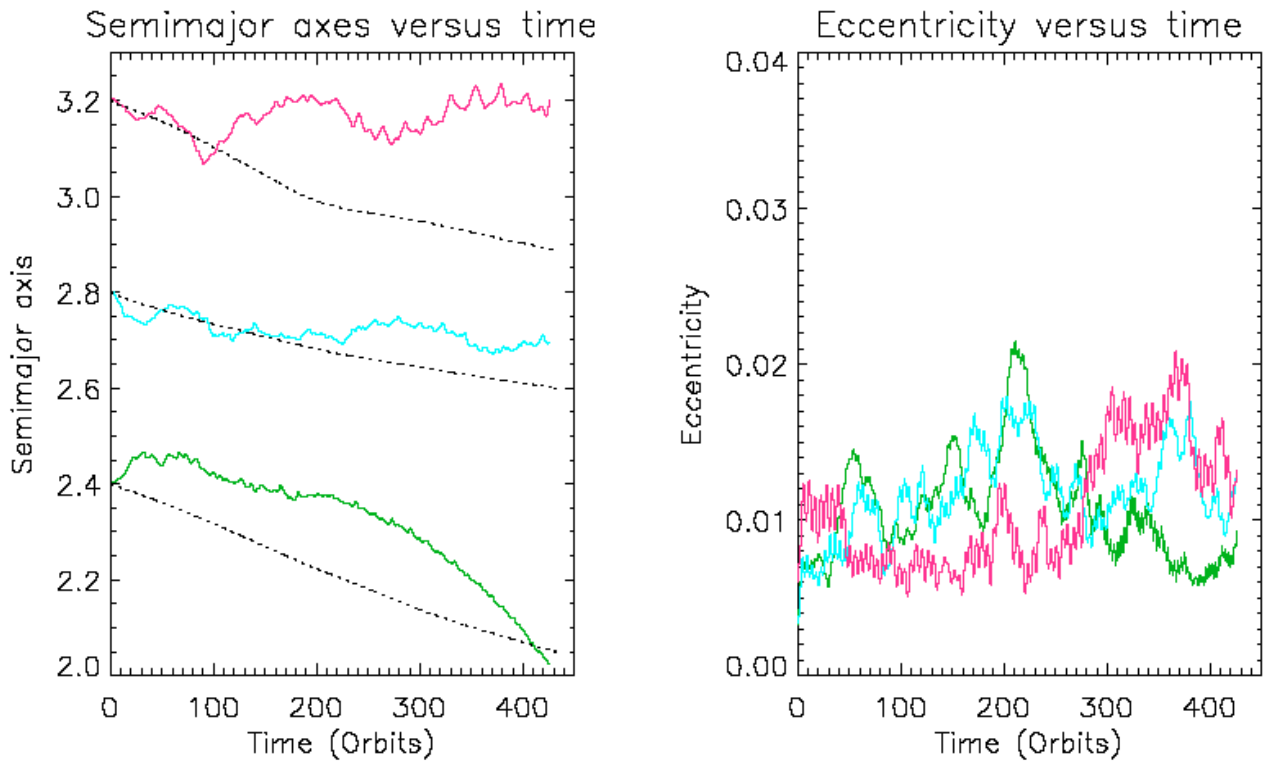

Fig. 10. This figure shows the evolution of the planet semimajor axes (left panel) and eccentricities (right panel) for the run with $m_{\mathrm{p}}=30 M_{\oplus}$. The figures show that the outer two planets undergo migration that is substantially different to the laminar disk runs. The inner planet migrates primarily inward as the disk turbulence is reduced in the inner regions. Note that the scale on the $y$ axis in the right panel differs from that of similar plots pertaining to lower mass protoplanets. 

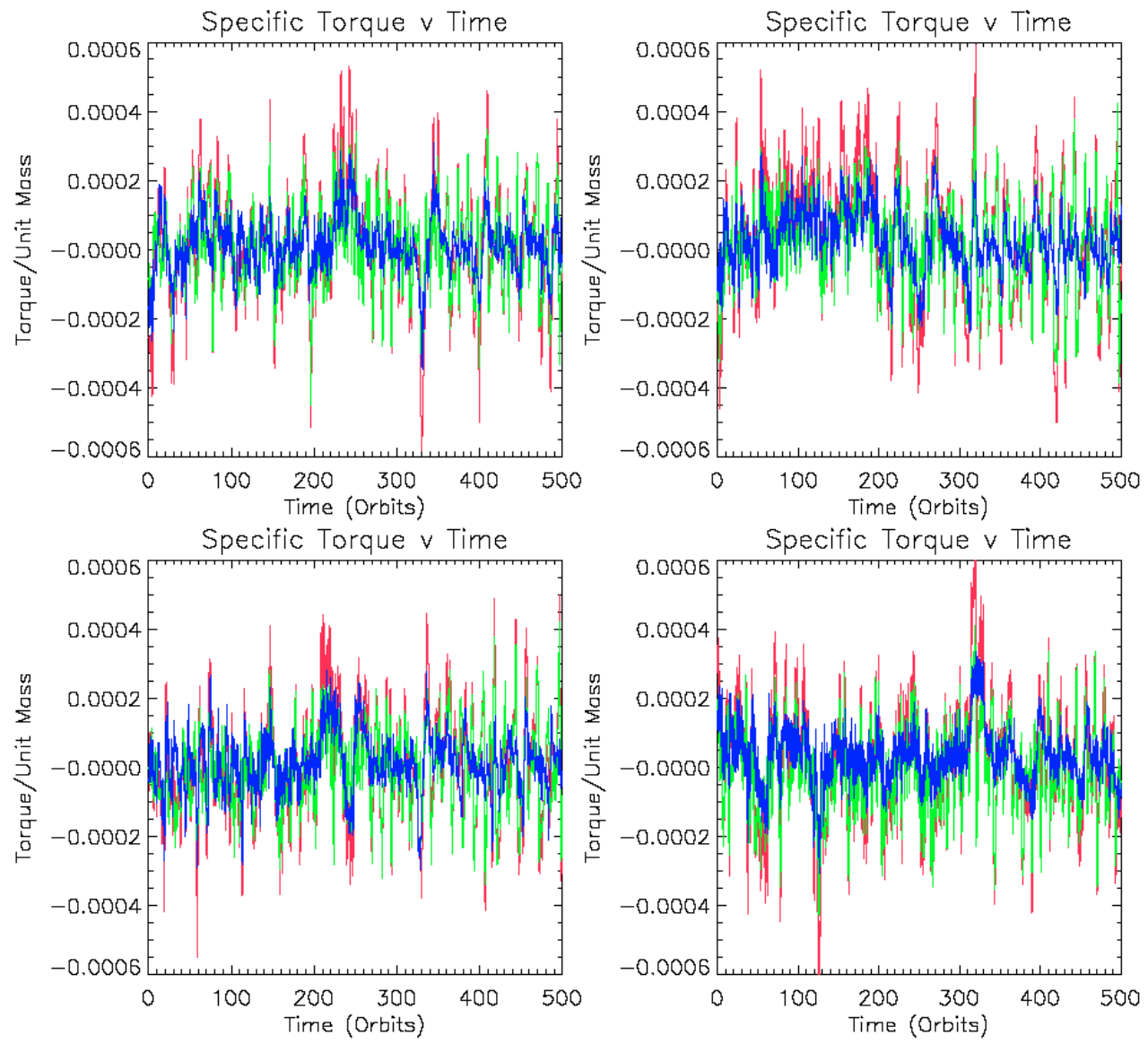

Fig. 11. This figure shows the time evolution of the torques per unit mass for four different planets selected from four different simulations. Moving from left to right and from top to bottom the planet masses and initial orbital radii are: $\left(m_{\mathrm{pi}}=0, r_{\mathrm{pi}}=2.6\right) ;\left(m_{\mathrm{pi}}=1, r_{\mathrm{pi}}=2.4\right)$; $\left(m_{\mathrm{pi}}=3, r_{\mathrm{pi}}=2.8\right) ;\left(m_{\mathrm{pi}}=10, r_{\mathrm{pi}}=2.6\right)$ The light grey (green) line corresponds to the torque exerted on the planet by the outer disk, the black (blue) line shows the torque due to the inner disk, and the dark grey (red) line shows the total torque. Moving from $m_{\mathrm{p}}=0$ to $m_{\mathrm{p}}=10 M_{\oplus}$ we observe that the inner and outer disk torques tend to separate, but that the torque fluctuations due to the turbulence remain significantly greater in amplitude than the mean torque.

show a significant deviation from the trajectories followed by planets in a laminar disk model. The middle planet undergoes noisy inward migration, but at a rate substantially less than that obtained for the laminar model. The outermost planet undergoes essentially no net migration for the duration of the model run time. It is clear that stochastic effects due to the turbulence continue to have an impact on the migration of $30 M_{\oplus}$ protoplanets.

The eccentricity evolution is shown in the right panel of Fig. 10. The values obtained are similar to those for the $10 M_{\oplus}$ planets, indicating little growth of the eccentricity because the damping induced by the underlying type I resonant disk interaction.

\section{Stochastic torques and type I migration}

We now examine the torques experienced by the protoplanets during the simulations in more detail. In view of the large number of planets considered, we restrict our discussion to a few specific examples which are illustrative of the range of behaviour observed in the simulations as a whole. We also discuss the implications for type I migration of low mass planets in turbulent disks.

\subsection{Time evolution of stochastic torques}

Figure 11 shows four examples of the time evolution of the torque per unit mass experienced by the planets in the simulations. The torque per unit mass is defined by Eq. (9), and is presented in the units described in Sect. 4. Moving from left to right and from top to bottom, the panels show results from simulations with: $\left(m_{\mathrm{pi}}=0, r_{\mathrm{pi}}=2.6\right) ;\left(m_{\mathrm{pi}}=1, r_{\mathrm{pi}}=2.4\right)$; $\left(m_{\mathrm{pi}}=3, r_{\mathrm{pi}}=2.8\right) ;\left(m_{\mathrm{pi}}=10, r_{\mathrm{pi}}=2.6\right)$, where the radii, $r_{\mathrm{pi}}$, refer to the initial orbital radii of the planets. In each panel, the torque on the planet due to the inner disk is shown by the black (blue) line, that due to the outer disk is shown by the light grey (green) line, and the dark grey (red) line represents the total torque. In all cases the torque is a highly variable quantity, as discussed previously in NP2004. On the same scale, the type I torque due to an equivalent laminar disk is $\simeq 1.5 \times 10^{-6}\left(\frac{m_{\mathrm{pi}}}{M_{\oplus}}\right)$. This value was obtained from the simulations of low mass planets embedded in laminar disks represented by the dotted lines in Figs. 5-8. Comparison with the migration time given by Eq. (32) of Papaloizou \& Larwood (2000) shows that our simulated torques are smaller by a factor of $\simeq 0.71$ compared to their fit to linear calculations. Stochastic variations relative to type I torques therefore typically range between 10 and 100 for planet 

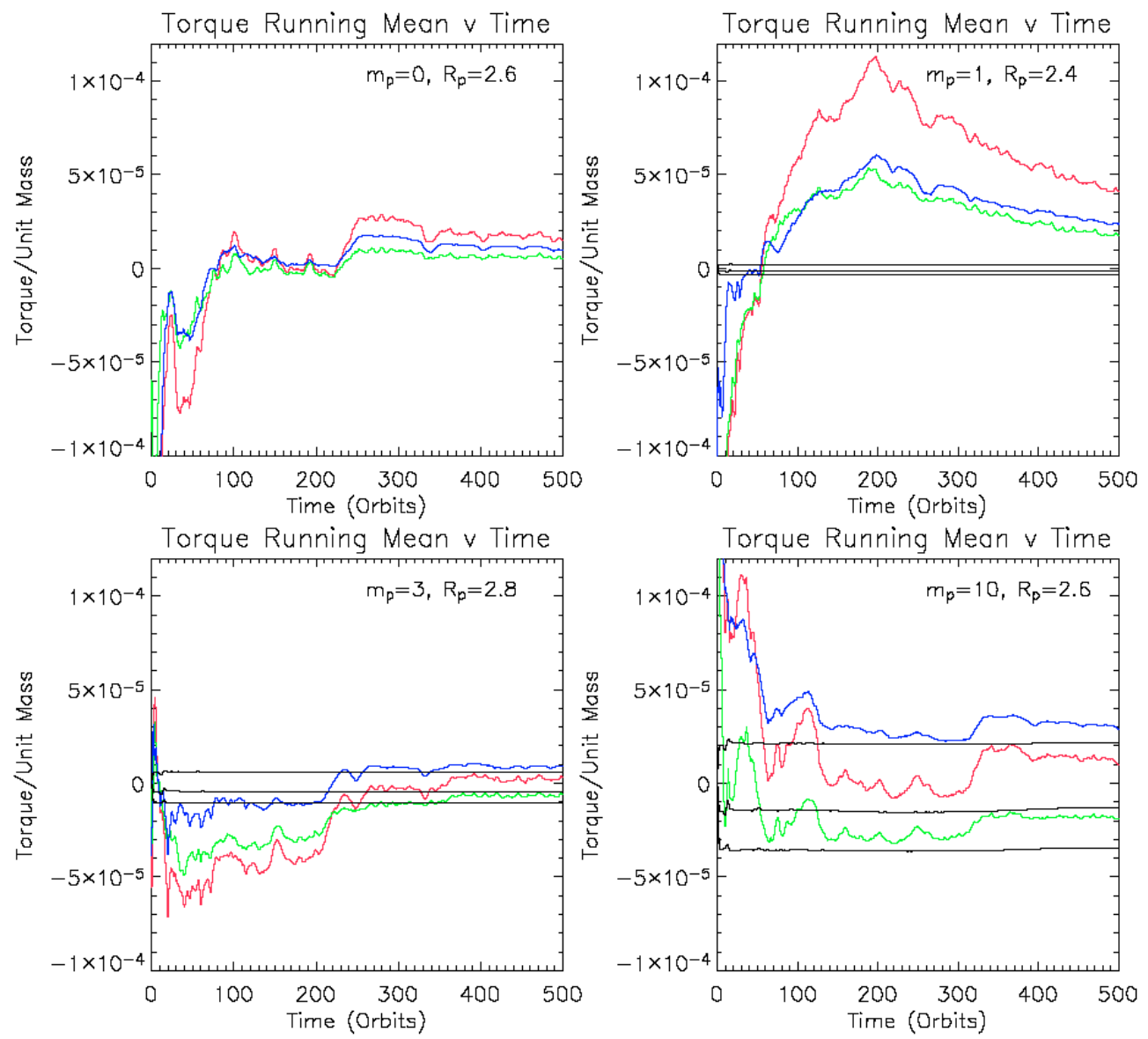

Fig. 12. This figure shows the time evolution of the running mean torques per unit mass for the four simulations shown in Fig. 11. The planet masses and initial orbital radii are indicated at the top right of each panel. The light grey (green) line corresponds to the torque exerted on the planet by the outer disk, the black (blue) line shows the torque due to the inner disk, and the dark grey (red) line shows the total torque. Also plotted with black lines are the running means obtained from equivalent simulations with viscous, laminar disk models. Here the upper line is due to the inner disk, the lower line is due to ther outer disk, and the middle line is the total torque.

masses in the range 1 to $10 M_{\oplus}$, but with peak fluctuations being up to four times larger than these values. As was noted in NP2004, these large fluctuations make it difficult to separate the torques due to the inner and outer disk when inspecting Fig. 11 for planet masses $m_{\mathrm{p}} \leq 10 M_{\oplus}$.

The time evolution of the running means of the torques displayed in Fig. 11 are shown in Fig. 12, along with the running means of the torques from the equivalent laminar disk simulations. In all cases, the running means do not converge to the equivalent type I laminar disk torques during the simulations, as one would expect from inspecting the migration histories presented in Figs. 4-8. If good convergence of the running means to the appropriate type I torque is possible, then these figures show that runs times considerably longer than those presented here will be required to achieve it. Such runs are currently not feasible computationally.

This latter point concerning convergence is illuminated to some degree by Fig. 13, which shows the power spectrum of the temporal evolution of the total torques plotted in Fig. 11. In these figures, a frequency of between $0.2-0.3$ represents the orbital periods of protoplanets located at orbital radii between $r_{\mathrm{pi}}=2.23-2.92$. The power spectra are obtained by Fourier analysis of the time evolution of the total torques generated during the simulations. These torques are output every ten time steps during the simulations, with the time interval between each output being $\Delta t$. If $N$ is the total number of data points then we can define discrete frequencies by

$\omega_{n}=\frac{2 \pi n}{N \Delta t}$

where $n=-N / 2, \ldots, N / 2$. The discrete Fourier transform of the torques is then

$H\left(\omega_{n}\right)=\frac{1}{N} \sum_{k=0}^{N-1} T_{k} \exp \left(\mathrm{i} \omega_{n} t_{k}\right)$

where $t_{k}$ is the time corresponding to data point $k$, and $T_{k}$ is the torque. We define an amplitude by

$A\left(\omega_{n}\right)=\sqrt{\left|H\left(\omega_{n}\right)\right|^{2}+\left|H\left(-\omega_{n}\right)\right|^{2}}$.

$A\left(\omega_{n}\right)$ is plotted against $\left|\omega_{n}\right|$ in Fig. 13.

The power spectra show that there is significant signal in the long term variation of the torques, with the total run time corresponding to a frequency of 0.002 in these plots. This explains why the averaged torques in Fig. 12 do not converge to 

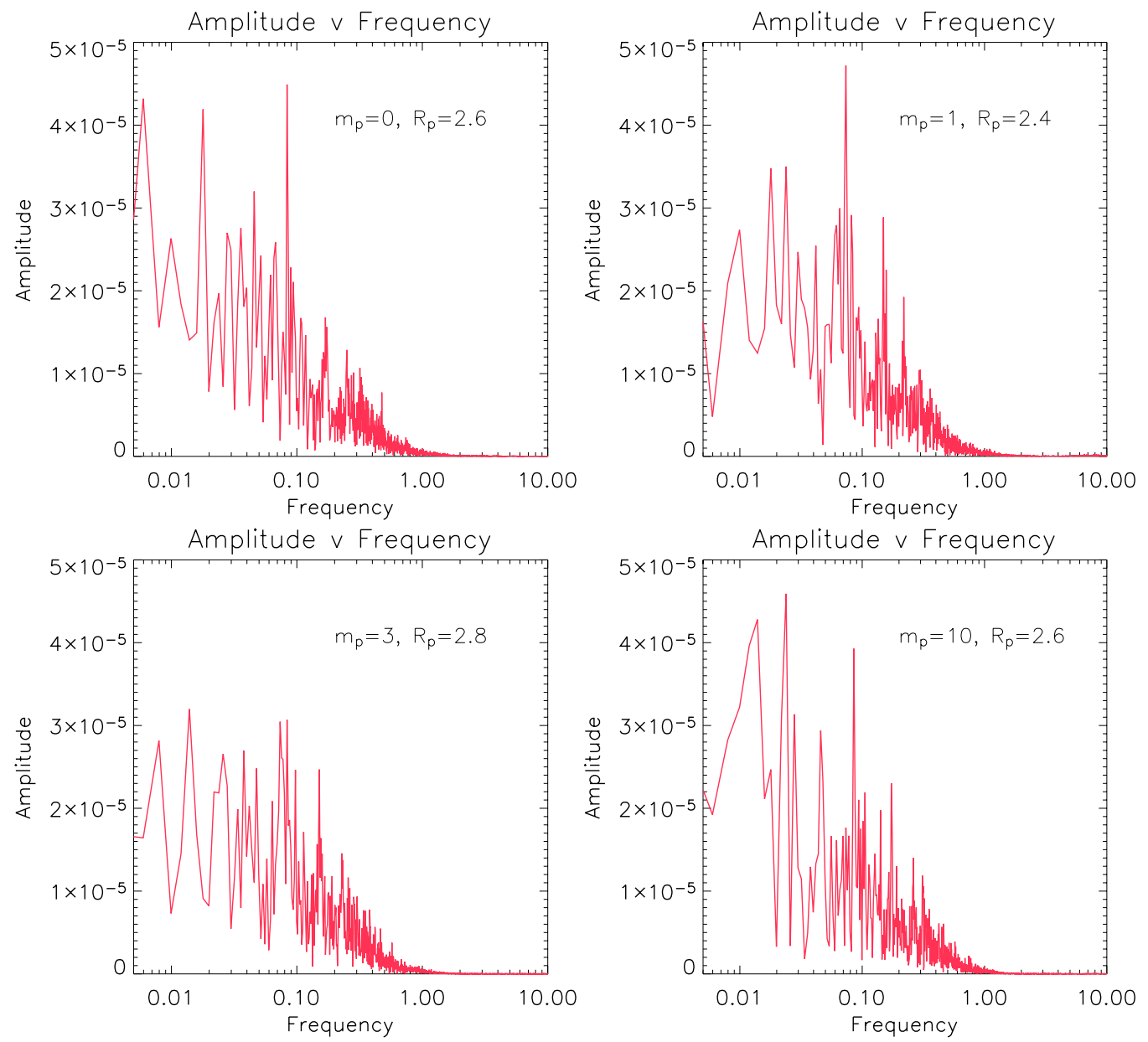

Fig. 13. This shows the power spectrum of the total torques plotted in Fig. 11. The main point to be taken from these plots is that significant power exists in frequencies that correspond to the longest time scales in the simulation, suggesting that low frequency variations in the torques experienced by the planets are important.

a well defined value: the stochastic torques contain contributions with significant amplitude whose associated time scale of variation is similar to the simulation run time.

The origin of these low frequency fluctuations is unclear. One possibility is that global communication across the disk on the viscous evolution time may lead to modification of the local turbulence on that time scale. This may in turn feed into the torque fluctuations experienced by embedded protoplanets. The disks we consider in this paper do not have a very large range in radii, but for the disk parameters $H / r=0.07$ and $\alpha=5 \times 10^{-3}$ the viscous communication time between the region where the planets are located (centred around $r=2.8$ ) and the disk outer edge $(r=4.5)$ is $\tau_{v} \simeq 8800$ orbits. Given that the simulations have been run for 500 orbits, communication between neighbouring regions of the disk may be influencing the turbulence on this time scale. One test of this would be to compare the power spectra of fluctuations in global disk calculations with local simulations performed in the shearing box. If the local simulations do not show the long term fluctuations then this could be taken as evidence that global communication is important. If low frequency fluctuations are observed, then it would indicate that these fluctuations are the property of the locally operating dynamo.
The torque frequency distributions corresponding to Fig. 11 are plotted in Fig. 14. These were calculated by sampling the torque experienced by the planets every 10 time steps during the simulations. The torques were then binned with bin widths $\Delta T=10^{-5}$, and the number in each bin counted. Although not strictly Gaussian, these distributions are similar to Gaussian profiles with standard deviation between $\sigma_{\mathrm{T}} \simeq 1-2 \times 10^{-4}$.

\subsection{Comparing stochastic migration and type I migration torques}

In a previous study, NP2004 considered the orbital evolution of low mass planets in turbulent disks, and treated the issue of torque convergence as a simple signal-to-noise problem. The basic assumption here is that the torque experienced by the planet, $T(t)$, consists of a linear superposition of a rapidly varying term, $T_{\mathrm{f}}(t)$, caused by turbulent fluctuations, and a constantly acting type I torque $\langle T\rangle$ :

$T(t)=\langle T\rangle+T_{\mathrm{f}}(t)$

Assuming that the stochastic torques are Gaussian distributed (the Central Limit Theorem tells us that even if the torques are not normally distributed, their cumulative effects will be similar 

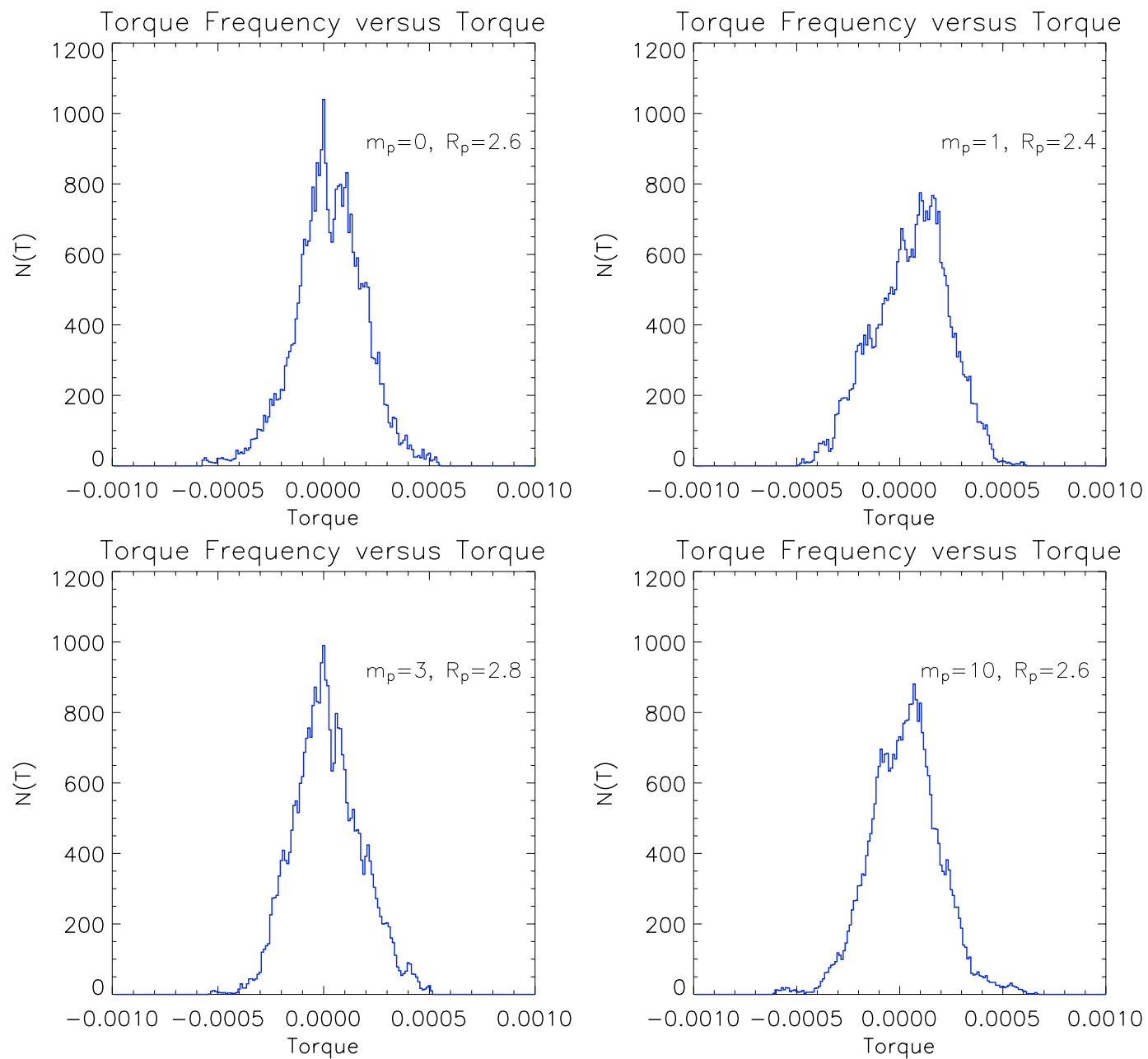

Fig. 14. This shows the frequency distribution of the torques plotted in Fig. 11. The plots show that the stochastic torques experienced by the planets have distributions that are fairly close to being Gaussian with standard deviation $\sigma(T) \simeq 1-2 \times 10^{-4}$.

to normally distributed torques), the time average of Eq. (20) becomes

$\bar{T}=\langle T\rangle+\frac{\sigma_{\mathrm{T}}}{\sqrt{t_{\mathrm{tot}}}}$.

Here $\sigma_{\mathrm{T}}$ is the standard deviation of the torque amplitude, and $t_{\text {tot }}$ is the total time elapsed, measured in units of the characteristic time for the torque amplitude to vary. Convergence of the torques toward the underlying type I value is expected to occur once the two terms on the right hand side become equal.

Using a very simple by-eye inspection of "torque versus time" plots, similar to Fig. 11, NP2004 estimated the time for torque variation and the typical amplitude of the fluctuating torques. Using these in Eq. (21) they derived a time for torque convergence of $\simeq 70$ planet orbits for a $10 M_{\oplus}$ protoplanet. Following a similar procedure, inspecting a blow-up of Fig. 12 leads to a by-eye estimate of the torque variation time $\simeq$ half a planet orbital period (the same as used by NP2004). Inspecting the torque distrbutions in Fig. 14 gives a value of the torque standard deviation $\sigma_{\mathrm{T}} \simeq 1.5 \times 10^{-4}$. Inserting these values into Eq. (21), and using a type I torque value of $1.5 \times 10^{-6}\left(\frac{m_{\mathrm{pl}}}{M_{\oplus}}\right)$, gives an estimated time for torque convergence equivalent to 50 planet orbital periods when the protoplanet mass is $10 M_{\oplus}$.
The modest difference in this estimate compared to NP2004 arises because the estimates of the standard deviation of torque fluctuations differ. For planets distributed in radius as they are in our simulations, this translates into a convergence time of between 163-286 orbits measured at $r=1$. This crude analysis predicts that we should see inward migration for the $10 M_{\oplus}$ planets in Fig. 8, which is clearly not the case.

Treating the issue of torque convergence using the above approach has a number of problems. The main one is illustrated by Fig. 13, which clearly demonstrates that there is no single frequency associated with the torque variation, and furthermore indicates that a simple by-eye examination of torque versus time plots can be misleading about which time scale is dominant. A more sophisticated treatment should take account of the fact the stochastic torques have significant amplitude distributed across a broad range of frequencies. We have taken a different approach to addressing the issue of torque convergence that ought to be more accurate.

In order to test whether the evolution of low mass planets in turbulent disks can be described as a superposition of stochastic torques and constantly acting type I torques, we have performed a series of $N$-body simulations in which particle orbits were evolved under the influence of prescribed forces. 
These included stochastic forces, which were calculated using a Fourier analysis of the migration torques obtained in the MHD simulation with planet masses $m_{\mathrm{pi}}=0$ (i.e. the "planetesimal" run shown in Fig. 4). This Fourier analysis allowed forces from the actual MHD simulations to be incorporated into simple integrations of particle orbits. The Fourier transform of the torques as a function of time is given by Eq. (18) in Sect. 7.1. The reconstructed torque at time $t$ is then given by the inverse transform:

$T(t)=\sum_{n=0}^{N-1} H\left(\omega_{n}\right) \exp \left(-\mathrm{i} \omega_{n} t\right)$.

This expression can be calculated for any value of $t$ required, and so provides a means of including the stochastic torques in an $N$-body simulation. We note, however, that the torques calculated during the MHD simulations were only output once every ten time steps, rather than at every time step. This means that our reconstructed torques are not exactly the same as the torques experienced by the planets during the MHD simulations. The variation of the torque experienced by a planet over ten time steps is not great (the torque shows significant variation over $\sim$ one thousand time steps), so we expect the reconstructed torques to be a good approximation to the real torques.

In addition to including stochastic force, type I torques taken from Eq. (32) of Papaloizou \& Larwood (2000) were included, for which the inward migration rate is linearly proportional to the planet mass. We note that comparing torques from laminar disk simulations, and those given in Papaloizou \& Larwood (2000), show that those obtained in the simulations are smaller by a factor of $\simeq 0.71$, so we multiplied the type I torques used in the $N$-body simulations by this factor.

The particle orbits were integrated using a simple leap-frog integrator. The time step used was held constant, and was chosen to be equal to the mean time step size which arises from the Courant condition in the MHD simulations. The time step calculated during the MHD simulations varied by only a few percent throughout the simulations.

The results of these runs are shown in Fig. 15, where the planet mass is indicated in the upper right corner of each panel. For zero-mass planets, orbit evolution similar to that shown in Fig. 4 was obtained. This indicates that the sampling of torques after every ten time steps in the MHD simulations leads to reconstructed torques that are quite accurate. For successively higher masses, these orbit trajectories were modified by having a small inward drift superposed. For planet masses $m_{\mathrm{pi}} \geq 10 M_{\oplus}$, a definite inward drift that overwhelms the stochastic migration can be observed. It is worth noting that the inward drift observed for the $10 M_{\oplus}$ cases in Fig. 15 is not matched by the actual MHD simulation presented in Fig. 8 .

We should point out that we have only sampled six realisations of the turbulence when performing the calculations presented in Fig. 15. On this basis alone it would be wrong to draw too strong a conclusion about the role of underlying type I torques in the MHD simulations. Inspection of Figs. 4-8, and Figs. 13 and 14 show that the torque distributions can differ substantially from run to run, such that the results shown in Fig. 15 may simply be a reflection of a minority of possible outcomes.
To test this further we ought to perform additional simulations of MHD turbulence with zero-mass test particles being used to sample the torque fluctuations. This, however, would be computationally expensive. The remaining simulations we have presented, for finite mass planets, contain the effects of turbulent fluctuations and spiral density waves (not always visible against the turbulent backdrop) excited by the planets. As such they cannot be used to sample cleanly the role of turbulence-induced fluctuations, but including them in the current analysis is still useful.

A $10 M_{\oplus}$ planet in a laminar disk migrates a distance of $\Delta r \simeq 0.15$ in 500 orbits (as shown by the dotted lines in Fig. 8). We now consider the effect of superposing such a drift on the migration histories of all planets plotted in Figs. 4-8 whose orbits started between radii $r_{\mathrm{pi}}=2.6-3.2$. Of this sample of twenty planets, a subset of eleven show net outward migration prior to the superposition. Of these eleven, only one would show net outward migration after superposition (this would be the $10 M_{\oplus}$ planet that was initiated at $r_{\mathrm{pi}}=3.0$ ), two would show no net migration, and eight would show a net inward drift. Based on this apparent tendency for the simulations as a whole to show inward migration when type I drift is superposed, which differs from the outcome observed in Fig. 8 for which the planet mass is $10 M_{\oplus}$, there is a hint that the effects of type I migration may be diminished in a turbulent disk. This may arise because of density and pressure perturbations in the vicinity of the planet modifying the excitation of spiral density waves and the usual bias between inner and outer disk. At present, however, the results of the simulations are sufficiently ambiguous for such a conclusion to be premature. It remains possible that the fluctuation spectrum associated with the orbital evolution shown in Fig. 8 is such that it is simply able to overcome the effects of type I migration for the duration of the simulations due to significant contributions from low frequency components. Resolution of these questions requires simulations to be run for longer duration, and for customised simulations to be performed that examine in detail the exchange of angular momentum between planet and turbulent disk. These calculations will be the subject of future publications.

\section{Eccentricity evolution}

In order to examine the origin of the behaviour of the orbital eccentricities observed in Figs. 4-8, we performed simulations similar to those described at the end of Sect. 7.2. Particle orbits were evolved using a simple leap-frog integrator, and with prescribed forces included representing stochastic eccentricity forcing and eccentricity damping associated with interaction at coorbital Lindblad resonances. The stochastic forces were calculated using a Fourier reconstruction of the radial and azimuthal forces experienced by the "planetesimals" in the MHD simulation for which the planet mass $m_{\mathrm{pi}}=0$, results for which are shown in Fig. 4. The eccentricity damping was included by using Eq. (38) of Papaloizou \& Larwood (2000). As already described, the source of this damping is primarily interaction with the disk at coorbital Lindblad resonances (e.g. Artymowicz 1993). 

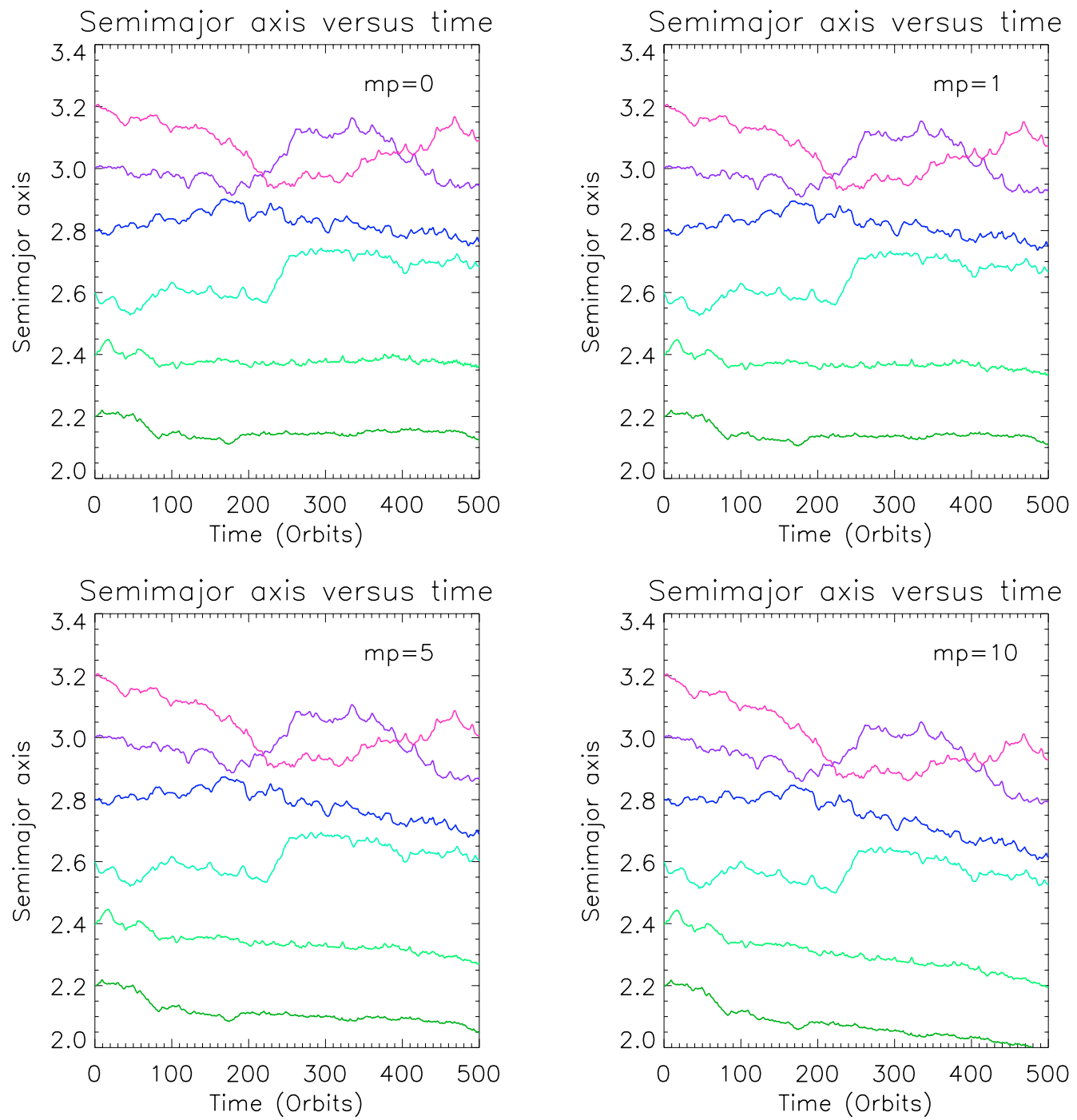

Fig. 15. This figure shows the evolution of semimajor axes from simulations in which the stochastic torques generated by the MHD simulation with $m_{\mathrm{p}}=0$ were reconstructed using Fourier analysis, and type I migration torques were included using a scaled version of Eq. (32) from Papaloizou \& Larwood (2000). For planet masses lower than $10 M_{\oplus}$, stochastic evolution of the orbits is dominant. For $m_{\mathrm{p}}=10 M_{\oplus}$, the effects of the superposed type I migration torques are dominant.

We calculated orbital evolution for planets with masses $m_{\mathrm{pi}}=0,1,3,5,10$, and $30 M_{\oplus}$ as in the MHD simulations. The masses simply control the degree of eccentricity damping, whereas the eccentricity driving is independent of mass. A selection of the resulting eccentricity behaviours are shown in Fig. 16. These figures show that good agreement is obtained with the evolutionary trends found in the full MHD simulations, suggesting that the eccentricity evolution obtained in those runs was indeed due to stochastic forcing by the turbulence, and damping due to resonant disk interaction. It is interesting to note that the agreement with the eccentricity evolution is better than that obtained when considering the superposition of type I torques and stochastic forces in Sect. 7.2. The reason for this is simply that the type I migration torques rely on there being an asymmetry between the inner and outer disk contributions, which may not occur as the density field near the planet varies erratically. The eccentricity damping, however, is largely produced by coorbital Lindblad torques located near the orbit of the planet. These should always be present throughout the MHD simulations, and operate as a constant source of damping, although the rate of damping may vary with time due to fluctuations induced in the disk structure by the turbulence.

\section{Discussion}

\subsection{Long term evolution of planetesimal orbits}

Here we consider only objects for which gravitational fluctuations due to the turbulent disk dominate the dynamics rather than gas-drag forces. Plantesimals with radius $1-10 \mathrm{~km}$ fall into this category. The migration histories of the simulated planetesimals plotted in Fig. 4 show that such bodies will undergo substantial migration via a random walk if protoplanetary disks are globally turbulent. The mean migration distance of the planetesimals in Fig. 4 is $\Delta r \simeq 0.09$ over a time of 500 orbits measured at $r=1$. If we make the simplifying 

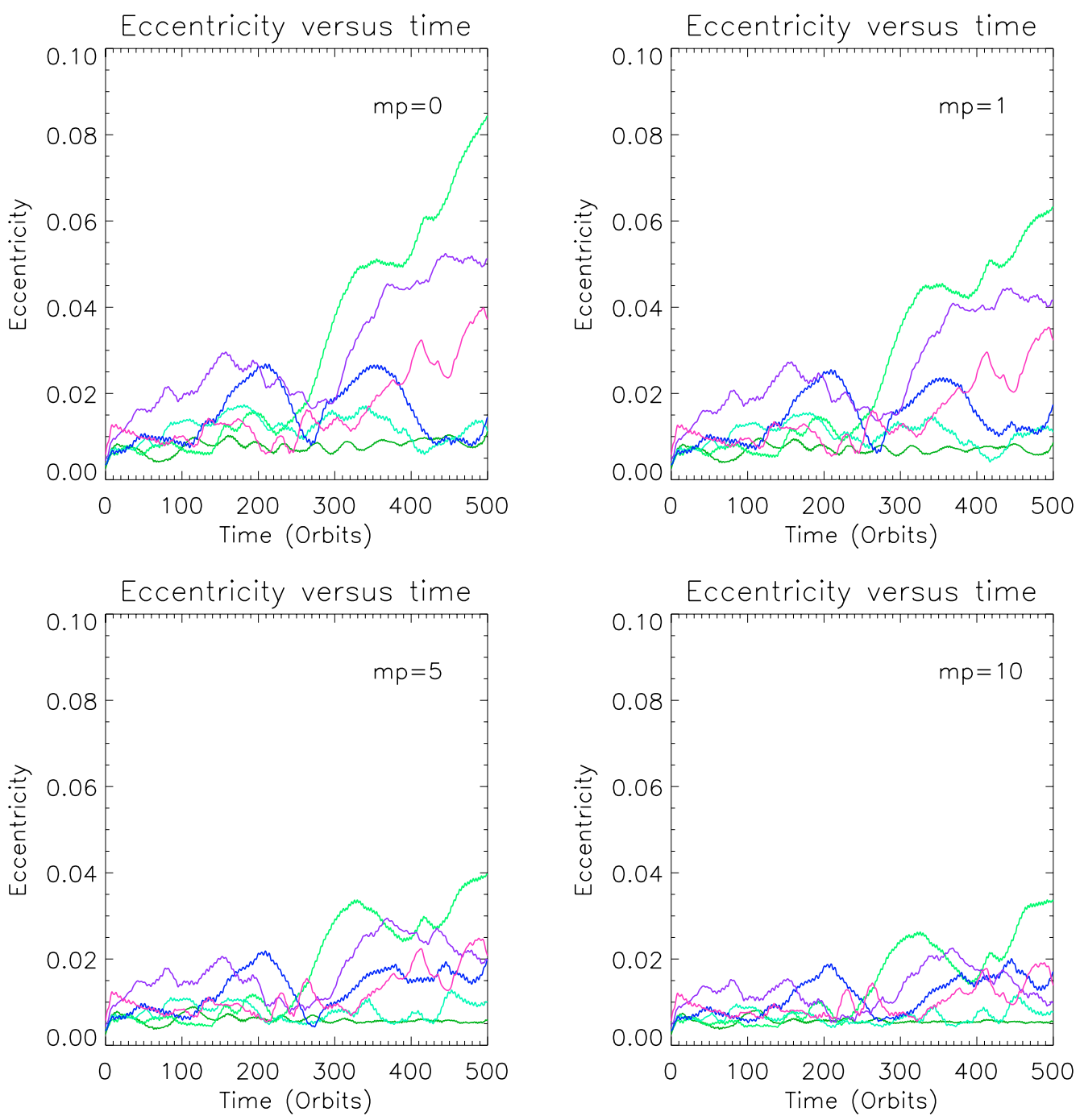

Fig. 16. This figure shows the evolution of eccentricities from simulations in which the stochastic torques generated by the MHD simulation with $m_{\mathrm{p}}=0$ were reconstructed using Fourier analysis, and eccentricity damping was included using Eq. (38) from Papaloizou \& Larwood (2000). The planet mass in each case is indicated in the top right of each panel. A clear trend in decreasing eccentricity may be observed as the masses of the planets increase, in broad agreement with the full MHD simulation results presented in Sects. 6.3-6.9.

assumption that the migration distance scales according to a random walk, so that $\Delta r \propto \sqrt{t}$, where $t$ is the time elapsed, and further take the location $r=2.5$ in the simulations to be equivalent to $5 \mathrm{AU}$, then the time taken for a typical planetesimal to migrate a distance equal to its semimajor axis is $t \simeq 1 \mathrm{Myr}$. The stochastic torques were generated by a disk model that is approximately three times more massive than a minimum mass model. A lower mass disk would generate a correspondingly longer migration time.

It is clear that planetesimals in turbulent disks will undergo significant migration during the lifetime of the nebula. From the point of view of forming planets quickly, turbulenceinduced changes to the orbital elements can have both a positive and a negative effect. Increased mobility generally acts to favour forming planets more quickly. One of the major issues facing the theory of planet formation is forming the cores of the giant planets fast enough to accrete gas before dispersal of the nebula. Although estimates of the core formation time based on models of runaway growth suggest relatively short core formation times (e.g. Pollack et al. 1996), simulations indicate that runaway growth does not continue all the way to the completion of core formation. Runaway growth slows down and enters a stage of "oligarchic growth" when the eccentricities and inclinations of the planetesimals are pumped up by the larger embryos (Ida \& Makino 1993; Kokubo \& Ida 1998). Simulations by Thommes et al. (2003) indicate that core formation becomes difficult, in part due to gap formation in the planetesimal disk by the larger embryos and in part due to the dynamical excitation of the planetesimal disk, leading to cores of $\sim$ few $M_{\oplus}$ forming in a few million years. The isolation of cores may be alleviated by the increased mobility of embryos and planetesimals in a stochastic migration scenario. In addition, the existence of regions of the disk where the turbulence is weaker than in others may lead to the accumulation of planetesimals there that could speed up accumulation processes.

The eccentricity and (and presumably) inclination driving by turbulence indicated by the right panel of Fig. 4, however, may slow down the early stages of planet formation. 
In particular the runaway growth of planetesimals will be affected, as the velocity dispersion of small bodies needs to be smaller than the escape velocity from the larger accreting objects. Indeed a large velocity dispersion generated by turbulence may lead to destructive rather than accumulative collisions between planetesimals, leading to questions about how planetesimals form at all in a turbulent disk. It should also be noted that the large scale migration of icy planetesimals from the outer solar system into the inner solar system may lead to the inner planets being more enriched in volatiles than is observed.

These problems may be alleviated if the stochastic torque values obtained in the MHD simulations performed here, using cylindrical disk models, turn out to be overestimates when models including vertical stratification are computed. A vertical gradient may then occur in the relative density fluctuations due to magnetic buoyancy effects (e.g. Stone et al. 1996; Stone $\&$ Miller 2000), which reduce the torque fluctuations at the disk midplane. The effects of a vertical gradient in the ionisation fraction (e.g. Gammie 1996) would act in a similar manner.

\subsection{Long term evolution of protoplanet orbits}

The situation regarding the long term evolution of planetesimals in turbulent disks is clear - they will slowly diffuse throughout the disk. The situation regarding high mass, gap forming planets also seems to be clear. They will migrate inward at the effective viscous evolution of time of the disk, as in the standard type II migration picture (Nelson \& Papaloizou 2003, 2004). The situation regarding low mass protoplanets remains ambiguous. The central question remains: can stochastic forces overcome type I migration and prevent at least some low mass protoplanets from falling into the star prior to accreting a gaseous envelope and becoming gas-giants?

The answer to this question depends on how effective type I torques are in a turbulent disk, and whether the disk turbulence can generate fluctuations with the required amplitudes and temporal behaviour to counterbalance the inward drift on planet formation time scales of $\sim 1$ Myr. The simulations presented in Sects. 6.3-6.9 and Sect. 7.2 indicate that type I torques may be diminished in turbulent disks compared with those that arise in laminar disks, but the evidence is far from conclusive. The power spectra presented in Fig. 13 show that the disk turbulence is capable of generating long term fluctuations with the appropriate amplitude to significantly affect type I migration, but there is no way of predicting whether such fluctuations can persist for time scales appropriate to planet formation itself. This question can only be answered by simulations whose run times are currently too long to be performed.

The origin of the longer time scale fluctuations observed in the simulations remains unknown. We have suggested that global communication across the disk on the viscous time scale may feed into the fluctuations, contributing to their long term temporal evolution. The viscous communication time from the outer edge to the planet forming region in our model disks is $\simeq 8000$ orbits. For naturally occuring disks, that form through the collapse of molecular clouds and have radii in excess of $100 \mathrm{AU}$, the viscous evolution time exceeds 1 Myr. It is not unreasonable to suppose that the nature of the turbulence in the planet forming region between 1-10 AU may be affected by global communication through the disk on this time scale. This may in turn feed into the torques experienced by forming protoplanets.

Other effects that may arise in naturally occuring disks are the formation of surface density depressions or "gaps". Simulations show that variations in the magnetic stresses occur radially throughout the disk, and persistence of these can lead to the formation of "gaps" (e.g. Hawley 2000; Steinacker $\&$ Papaloizou 2002). Such features may play an important role in modifying the migration of low mass planets. The tendency of MHD turbulence to assist in gap formation for more massive planets has already been noted by Nelson \& Papaloizou (2003) and Winters et al. (2003). Radial variations in the strength of the disk turbulence may also play a role. The existence of "dead zones" has been suggested, particularly in the planet forming region around 5 AU (e.g. Gammie 1996; Fromang et al. 2002), with an inner active zone maintained through thermal ionisation of alkalii metals. A dominant outward flux of waves excited by a more active interior disk may provide a bias that helps stochastic torques counter balance inward type I migration.

\section{Conclusions}

In this paper we have presented simulations of low mass protoplanets embedded in turbulent, magnetised disks. The planet orbits are evolved, with forces due to gravitational interaction with the disk being included. The disk models are cylindrical, as the vertical component of gravity is neglected, but are otherwise designed to be similar to disks thought to be present around young stars during the planet formation epoch (i.e. disk thickness $H / r=0.07$; disk viscosity $\alpha \sim 5 \times 10^{-3}$ ).

Planet masses, $m_{\mathrm{p}}$, ranging between zero and $30 M_{\oplus}$ were considered. The $m_{\mathrm{p}}=0$ "planetesimals" were found to migrate through the disk in a manner similar to a random walk, and in some cases their eccentricity was found to increase to values $e \simeq 0.1$. The random walk element of their evolution provides a means of maintaining significant mobility of the planetesimal swarm during planet formation. This may help reduce the problems of orbital repulsion and core isolation found during simulations of giant planet core formation (e.g. Thommes et al. 2003). However, the increase in eccentricity may present a problem during the runaway growth stage of planet formation, which requires a dynamically cold disk of planetesimals (e.g. Wetherill \& Stewart 1993). Further simulations that include gas drag and vertically stratified disk models are required to obtain a more accurate picture of this eccentricity growth.

The protoplanets with finite masses that were studied also showed evidence of stochastic migration and eccentricity growth. The eccentricity evolution was such that the protoplanets with mass $m_{\mathrm{p}}=1$ and $3 M_{\oplus}$ showed peak eccentricities of $e \simeq 0.08$ and 0.045 , respectively, whereas the $m_{\mathrm{p}}=10$ and $30 M_{\oplus}$ planets had peak eccentricities of $e \simeq 0.02$. This is in broad agreement with expectations, as the interaction of low mass protoplanets with disks causes eccentricity damping due 
to interaction with material at coorbital Lindblad resonances (e.g. Artymowicz 1993). This damping is expected to scale linearly with the protoplanet mass, leading to the observed behaviour.

For all protoplanet masses in the range $1 \leq m_{\mathrm{p}} \leq 30 M_{\oplus}$, there was clear evidence of stochastic torques being dominant over the type I migration expected to occur in laminar disks. We considered an ensemble of models, and while some planets showed a definite trend toward inward migration, there were examples for each mass considered for which stochastic migration was the dominant effect. This behaviour arises in part because the turbulent fluctuations contain low frequency components whose amplitudes are such that they can significantly modify type I migration over the simulation run times that are currently feasible. In addition, we analysed the effect of superposing type I migration expected for laminar disks onto the stochastic migration obtained in turbulent disks. This analysis suggested that inward migration ought to be more apparent in the MHD simulations than was found to be the case, providing a hint that type I migration may be modified in turbulent disks. We note, however, that the simulations are not conclusive on this latter point.

A number of important issues remain to be addressed. These include a determination of the role of gas drag in modulating the effects of eccentricity growth of planetesimals, and the effects of vertical disk structure in influencing the strength of the stochastic torques at the disk midplane. The question of whether stochastic torques can prevent type I migration of protoplanets over planet formation time scales still remains to be settled, and can be partially answered by performing longer simulations. The potential modification of type I migration in turbulent disks can be addressed by customised simulations that examine the exchange of angular momentum between disk and planet. These will be the subjects of future publications.

Acknowledgements. The computations reported here were performed using the UK Astrophysical Fluids Facility (UKAFF) and the QMUL HPC facility purchased under the SRIF initiative. I would like to thank Richard Frewin, Alex Martin, and David Burgess for assistance with the computations presented here. Comments received from an anonymous referee have led to significant improvements in this paper.

\section{References}

Armitage, P. J. 1998, ApJ, 501, L189

Artymowicz, P. 1993, ApJ, 419, 166

Balbus, S. A., \& Hawley, J. F. 1991, ApJ, 376, 214

Bodenheimer, P., \& Pollack, J. B. 1986, Icarus, 67, 391

Boss, A. P. 2001, ApJ, 563, 367

Brandenburg, A., Nordlund, Å., Stein, R. F., \& Torkelsson, U. 1996, ApJ, 458, L45
D’Angelo, G., Henning, T., \& Kley, W. 2002, A\&A, 385, 647

Gammie, C. F. 1996, ApJ, 457,355

Goldreich, P., \& Tremaine, S. 1979, ApJ, 233, 857

Goldreich, P., \& Tremaine, S. 1980, ApJ, 241, 425

Hartmann, L., Calvet, N., Gullbring, E., \& D’Alessio, P. 1998, ApJ, 495,385

Hawley, J. F. 2000, ApJ, 528, 462

Hawley, J. F. 2001, ApJ, 554, 534

Hawley, J. F., \& Balbus, S. A. 1991, ApJ, 376, 223

Hawley, J. F., Gammie, C. F., \& Balbus, S. A. 1996, ApJ, 464, 690

Hawley, J. F., \& Stone, J. M. 1995, Comput. Phys. Commun., 89, 127

Ida, S., \& Makino, J. 1993, Icarus, 106, 210

Kley, W. 1999, MNRAS, 303, 696

Kokubo, E., \& Ida, S. 1998, Icarus, 131, 171

Lin, D. N. C., \& Papaloizou, J. C. B. 1979, MNRAS, 186, 799

Lin, D. N. C., \& Papaloizou, J. C. B. 1986, ApJ, 309, 846

Marcy, G. W., Cochran, W. D., \& Mayor, M. 2000, Protostars and Planets IV, ed. V. Mannings, A. P. Boss, \& S. S. Russell (Book Tucson: University of Arizona Press), 1285

Mayor, M., \& Queloz, D. 1995, Nature, 378, 355

Miller, K. A., \& Stone, J. M. 2000, ApJ, 534, 398

Nelson, R. P., \& Papaloizou, J. C. B. 2003, MNRAS, 339, 993 (NP2003)

Nelson, R. P., \& Papaloizou, J. C. B. 2004, MNRAS, 350, 849 (NP2004)

Nelson, R. P., Papaloizou, J. C. B., Masset, F., \& Kley, W. 2000, MNRAS, 318, 18

Papaloizou, J. C. B., \& Lin, D. N. C. 1984, ApJ, 285, 818

Papaloizou, J. C. B., \& Larwood, J. D. 2000, MNRAS, 315, 823

Papaloizou, J. C. B., \& Nelson, R. P. 2003, MNRAS, 339, 983 (PN2003)

Papaloizou, J. C. B., Nelson, R. P., \& Snellgrove, M. D. 2004, MNRAS, 350, 829 (PNS2004)

Papaloizou, J. C. B., \& Nelson, R. P. 2005, A\&A, 433, 247

Pollack, J. B., Hubickyj, O., Bodenheimer, P., et al. 1996, Icarus, 124, 62

Press, W. H., Teukolsky, S. A., Vetterling, W. T., \& Flannery, B. P. 1992, Numerical Recipes in Fortran: The Art of Scientific Computing (Cambridge: University Press)

Santos, N. C., Israelian, G., Mayor, M., Rebolo, R., \& Udry, S. 2003, A\&A, 398, 363

Shakura, N. I., \& Sunyaev, R. A. 1973, A\&A, 24, 337

Steinacker, A., \& Papaloizou, J. C. B. 2002, ApJ, 571, 413

Stone, J. M., Hawley, J. F., Gammie, C. F., \& Balbus, S. A. 1996, ApJ, 463,656

Tanaka, H., Takeuchi, T., \& Ward, W. R. 2002, ApJ, 565, 1257

Thommes, E. W., Duncan, M. J., \& Levison. H. F. 2003, Icarus, 161, 431

Van Leer, B. 1977, J. Comp. Phys., 23, 276

Vogt, S. S., Butler, R. P., Marcy, G. W., et al. 2002, ApJ, 568, 352

Ward, W. R. 1986, Icarus, 67, 164

Ward, W. R. 1997, Icarus, 126, 261

Wetherill, G. W., \& Stewart, G. R. 1993, Icarus, 106, 190

Winters, W., Balbus, S., \& Hawley, J. 2003, ApJ, 589, 543

Ziegler U., \& Yorke H. W. 1997, Comp. Phys. Comp., 101, 54 